\title{
Cover Semantics for Quantified Lax Logic
}

\author{
Robert Goldblatt \\ Centre for Logic, Language and Computation \\ Victoria University of Wellington, New Zealand \\ Rob.Goldblatt@msor . vuw.ac.nz
}

22 July, 2010

\begin{abstract}
Lax modalities occur in intuitionistic logics concerned with hardware verification, the computational lambda calculus, and access control in secure systems. They also encapsulate the logic of Lawvere-Tierney-Grothendieck topologies on topoi. This paper provides a complete semantics for quantified lax logic by combining the Beth-Kripke-Joyal cover semantics for first-order intuitionistic logic with the classical relational semantics for a "diamond" modality. The main technique used is the lifting of a multiplicative closure operator (nucleus) from a Heyting algebra to its MacNeille completion, and the representation of an arbitrary locale as the lattice of "propositions" of a suitable cover system. In addition, the theory is worked out for certain constructive versions of the classical logics K and S4.

An alternative completeness proof is given for (non-modal) first-order intuitionistic logic itself with respect to the cover semantics, using a simple and explicit Henkin-style construction of a characteristic model whose points are principal theories rather than prime saturated ones.

The paper provides further evidence that there is more to intuitionistic modal logic than the generalisation of properties of boxes and diamonds from Boolean modal logic.
\end{abstract}

\section{The Curious Concept of Lax Modality}

Propositional lax logic (PLL) was defined by Fairtlough and Mendler $[13,14]$ as an intuitionistic propositional logic with a modality $\bigcirc$ having the axioms

$$
\begin{aligned}
& \varphi \rightarrow \bigcirc \varphi \\
& \bigcirc \bigcirc \varphi \rightarrow \bigcirc \varphi \\
& \bigcirc \varphi \wedge \bigcirc \psi \rightarrow \bigcirc(\varphi \wedge \psi) .
\end{aligned}
$$

The motivation was hardware specification and verification, with a modal formula $\bigcirc \varphi$ being read "there is some constraint under which $\varphi$ is true". Typical constraints are timing delays on input signals to digital circuits, with the first axiom corresponding to a single wire with no delay; the second corresponding to the sequential composition of circuits, with constraint given by adding their delays; and the third axiom corresponding to parallel composition, with the maximum of the two delays as constraint. The term "lax" was chosen "to indicate the looseness associated with the notion of correctness up to constraints" $[14$, p. 3].

In fact there have been several independently motivated investigations that have produced an intuitionistic logic with a lax modality, i.e. one having the above axioms. ${ }^{1}$ The first would appear to be a Gentzen-style calculus studied by Curry [9] for proof-theoretic purposes. Its modality, denoted $\diamond$, was intended to express possibility.

Algebraically, a lax modality is modelled on a Heyting algebra by a multiplicative closure operator. Such an operator, known as a nucleus in locale theory [27], has the same formal properties as a LawvereTierney "topology" $j: \Omega \rightarrow \Omega$ on the object $\Omega$ of truth values in an elementary topos. This generalised the notion of a Grothendieck topology for presheaf topoi, and led Lawvere to suggest that the notion "appears

\footnotetext{
${ }^{1}$ These studies are also reviewed in Section 7.6 of the author's historical survey [23].
} 
most naturally as a modal operator of the nature 'it is locally the case that' " [33]. That perspective was investigated by the author in [20], which studied a system, equivalent to PLL, whose modality was denoted $\nabla$. It was provided with a complete semantics by taking Kripke's model theory for propositional intuitionistic logic [29] and adding a binary relation $R$ to interpret $\nabla$. The truth/satisfaction relation $x \models \nabla \varphi$ for the formula $\nabla \varphi$ at world/state $x$ was defined to hold iff

$$
\text { for all } y \text { such that } x R y, y \models \varphi \text {, }
$$

with some elementary conditions on $R$ imposed to validate the axioms for $\nabla$.

Notice that (1.1) is exactly the condition for satisfaction of a "box" modality $\square$ in Boolean modal logic. Classically, the deductive criterion for $\square$ to be a "Boolean box" is the derivability of the schemas

$$
\square \varphi \wedge \square \psi \rightarrow \square(\varphi \wedge \psi)
$$

and the monotonicity inference rule

$$
\frac{\varphi \rightarrow \psi}{\square \varphi \rightarrow \square \psi}
$$

This criterion ${ }^{2}$ is fulfilled by PLL when $\square$ is taken to be $\bigcirc$, but of course the underlying propositional logic of PLL is intuitionistic, not Boolean. The semantics of [20] presents a lax modality as a natural generalisation of a Boolean box to an intuitionistic context.

Because of the notation used for Lawvere-Tierney-Grothendieck topologies, a nucleus is sometimes called a " $j$-operator", or "J-operator" as in Fourman and Scott's extensive study [16]. Aczel [2] showed that a lax modality $\mathrm{J} \varphi$ can be defined in second-order intuitionistic logic as the formula $\forall p((\varphi \rightarrow p) \rightarrow p)$, where $p$ is a propositional variable. He called this the "Russell-Prawitz modality", because of its relation to certain definitions of $\wedge, \vee, \neg$ and $\exists$ in terms of $\rightarrow$ and $\forall$ that were introduced by Russell and studied by Prawitz in the intuitionistic setting. Aczel showed that any lax modality can be used to give a translation of intuitionistic logic into itself that generalises both the double negation interpretation and the RussellPrawitz representation. Concerning double negation, putting $\mathrm{J} \varphi=\neg \neg \varphi$ defines a lax modality over intuitionistic logic that Lawvere suggested should be read "it is cofinally the case that $\varphi$ " (see [20, p. 141] for an analysis of this).

The logical approach to topos theory of Bell [4] employs a lax modality, referred to as a "possibility operator", to construct categories of sheaves, using a "universal" closure operator on sets that is defined by this modality.

In a computational context again, Benton, Bierman and de Paiva [7] introduced a system called "CL-logic", equivalent to PLL, having a "curious possibility-like modality $\diamond "$. For a Hilbert-style axiomatisation, the axioms for $\diamond$ were given as

$$
\begin{aligned}
& \diamond \varphi \rightarrow((\varphi \rightarrow \diamond \psi) \rightarrow \diamond \psi) \\
& \varphi \rightarrow \diamond \varphi .
\end{aligned}
$$

CL-logic corresponds to the computational lambda calculus of Moggi [37], which has a type-constructor $T$ such that the denotation of a program computing values of type $A$ is itself of type $T A$. Categorically, $T$ is a "strong monad" on a cartesian closed category, and has the formal properties of a multiplicative closure operator.

Most recently, lax modalities have been used in access-control logics, dealing with authorisations in decentralised systems in which agents requested access to secure resources under the control of other agents $[17,18]$. These are multimodal logics, with lax modalities $\langle K\rangle$ indexed by agents $K$. A formula $\langle K\rangle \varphi$ is read " $K$ says $\varphi$ ", meaning that $K$ states that $\varphi$ is true. For example, $K$ may be a system administrator certifying that some other agent is an authorised user, or has access to a particular file. The $\langle K\rangle$ notation comes from dynamic logic, where it was designed to be reminiscent of the diamond symbol $\diamond$.

So although a lax modality has the defining deductive characteristics of a Boolean box modality, and is complete for the box-like semantics of (1.1), several authors have viewed it as a kind of "possibilistic" diamond. This may be because the axiom $\varphi \rightarrow \bigcirc \varphi$ is one of the first principles one thinks of when

\footnotetext{
${ }^{2}$ There are other equivalent criteria, most commonly the schema $\square(\varphi \rightarrow \psi) \rightarrow(\square \varphi \rightarrow \square \psi)$ together with the inference rule $\varphi / \square \varphi-$ see Section 7 .
} 
reading $\bigcirc$ as "possibly". Now the standard semantics for a Boolean diamond defines $x \models \diamond \varphi$ by the existential condition

$$
\text { there is some } y \text { such that } x R y \text { and } y=\varphi \text {, }
$$

dual to (1.1). In the presence of the standard semantics for disjunction, namely

$$
x \models \varphi \vee \psi \text { iff } x \models \varphi \text { or } x \models \psi,
$$

(1.2) ensures the validity of the axioms

$$
\begin{aligned}
& \diamond(\varphi \vee \psi) \leftrightarrow \diamond \psi \vee \diamond \psi \\
& \neg \diamond \perp,
\end{aligned}
$$

which are deductively characteristic of a Boolean diamond (here $\perp$ is a constant denoting a proposition that implies all others). But these axioms are not derivable in PLL when $\diamond$ is taken to be $\bigcirc$, so in that sense $\bigcirc$ is not diamond-like, and we cannot model the logic by combining (1.3) for disjunction with (1.2) as the definition of $x \models \bigcirc \varphi$. However there is clearly an existential aspect to the nature of $\bigcirc$ under the reading "there is some constraint ...", and this is reflected in the relational semantics provided for PLL in $[13,14]$, and extended to its first-order version QLL in [12]. Taking the non-modal part of QLL to be modelled by Kripke's semantics for first-order intuitionistic logic, ${ }^{3}$ the definition of $x \models \bigcirc \varphi$ is given as

$$
\text { for all } z \geq x \text { there is some } y \text { such that } z R y \text { and } y=\varphi \text {. }
$$

Here $\geq$ is the partial ordering of states used to interpret intuitionistic implication. So either of the conditions (1.1) and (1.5) can be used to semantically characterise a lax modality over the Kripkean intuitionistic semantics (with different extra conditions on $R$ in each case).

The main point of the present paper is to give another twist to this tale, by showing that the purely existential condition (1.2) can be used to model a lax modality after all, without validating (1.4) - provided we give up the classical semantics (1.3) for disjunction. In its place we adopt an interpretation of the non-modal connectives and the quantifiers that uses the cover-system structures involved in Grothendieck topologies. This approach, due to Joyal (cf. [28, Section 1]), is sometimes called "Kripke-Joyal semantics" because its treatment of conjunction, implication and the universal quantifier is reminiscent of Kripke's intuitionistic semantics. But it treats disjunction and the existential quantifier in a non-classical way that is reminiscent of the semantics of Beth [8], and indeed Beth's models are explicitly shown to be a special case of this approach in [6]. For simplicity we will refer to it just as cover semantics.

Accounts of this kind of semantics in topoi can be found in [26, 5.4], [31, II.8], [4, pp. 155ff] and [32, VI.6]. The history of Beth's own contribution is discussed in [44].

The basic idea of cover semantics comes from the topological notion of local truth. An assertion concerning an object $X$, which may be a space or an open subset of a space, is locally true if $X$ has a covering by open sets of each of which the assertion is true. For example, a function $f$ is locally constant on $X$ (i.e. the statement " $f$ is constant" is locally true of $X$ ) if $X$ has a covering of open sets on each of which $f$ is constant. Similarly, two such functions $f, g$ are locally equal on $X$ if $X$ has a covering of open sets on each of which $f$ and $g$ agree. (See [24], especially Chapter 14, as well as [20], for further discussion.)

Our abstract models for QLL will assign to each of their members $x$ a family of sets of members called the covers of $x$. We write $C \triangleright x$, or $x \triangleleft C$, to indicate that $C$ is a cover of $x$, or $x$ is covered by $C$. A condition is locally true of $x$ if there is some $C$ such that $C \triangleright x$ and each member of $C$ satisfies the condition, i.e. if $x$ is covered by a set of members that have this condition. We also use the notation $|\varphi|$ for the truth set of a sentence $\varphi$, i.e. the set $\{x: x \models \varphi\}$ of members at which $\varphi$ is true/satisfied. Then the truth relation $x \models \varphi \vee \psi$ for disjunction in cover semantics is defined to hold iff

$$
\text { there is some } C \triangleright x \text { with } C \subseteq|\varphi| \cup|\psi| \text {. }
$$

Now if $C \subseteq|\varphi| \cup|\psi|$, then each member of $C$ satisfies either $\varphi$ or $\psi$, so in any case satisfies the classical disjunction of $\varphi$ and $\psi$ in accordance with (1.3). Thus (1.6) tells us that $\varphi \vee \psi$ is true at $x$ in the cover semantics when the classical disjunction of $\varphi$ and $\psi$ is locally true at $x$.

\footnotetext{
${ }^{3}$ Except for the introduction of "fallible" worlds satisfying $\perp-$ see later in this section, and Remark 5.2.
} 
Our QLL-models each have a single domain $U$ over which the individual variables range. This contrasts with Kripke's models, where each state $x$ has its own domain $U_{x}$, and these domains expand along the partial ordering. In the cover semantics, the truth relation $x \models \exists v \varphi$ for the existential quantifier holds iff

$$
\text { there is some } C \triangleright x \text { with } C \subseteq \bigcup_{c \in U}|\varphi(c / v)| \text {. }
$$

In classical Tarskian semantics (and in Kripke's intuitionistic models), satisfaction of any instantiation $\varphi(c / v)$ is enough to ensure that $\exists v \varphi$ is true. Thus (1.7) tells us that $\exists v \varphi$ is true at $x$ in the cover semantics when the corresponding classical existential quantification of $\varphi$ is locally true at $x$.

The cover semantics criterion for $x \models \forall v \varphi$ is the classical Tarskian

$$
\text { for all } c \in U, x \models \varphi(c / v) \text {, }
$$

by contrast with Kripke's

$$
\text { for all } y \geq x \text { and all } c \in U_{y}, y \models \varphi(c / v) \text {. }
$$

Truth in our models is required to be a property of local character, or more briefly a local property, meaning one whose satisfaction is implied by its own local satisfaction. An illustration of this notion from above is the property of equality of functions: two locally equal functions are in fact equal, so equality is a local property. By contrast, a function can be locally constant on $X$ without being constant, so constancy is not a property of local character. Now if

$$
\text { there is some } C \triangleright x \text { with } C \subseteq|\varphi| \text {, }
$$

then $\varphi$ is locally true at $x$, in the sense that $x$ has a cover consisting of points at which $\varphi$ is true. We require that local truth implies truth, i.e. that if (1.8) holds, then $x=\varphi$ (cf. (5.2) below). This property is needed to ensure the validity of certain intuitionistic axioms (cf. Theorem 5.5). We express it by saying that $|\varphi|$ is a localised set. More generally, for any set $X$ define

$$
j X=\{x: \exists C(x \triangleleft C \subseteq X)\} .
$$

A member $x$ of $j X$ is a local member of $X$, because it is covered by a set of members of $X$. $X$ is localised if $j X \subseteq X$, meaning that all its local members are actual members, and hence that membership of $X$ is a property of local character. We require that each truth set $|\varphi|$ be localised.

It is also required that each truth set be an up-set, i.e. be closed upward under the partial order $\geq$ on models, as in the Kripkean semantics. A localised up-set is called a proposition, and the axioms we adopt for the covering relation $\triangleright$ are designed to ensure that the set Prop of propositions of a model is a complete Heyting algebra.

A given binary relation $R$ induces an operator $\langle R\rangle$ on subsets of a model by putting

$$
\langle R\rangle X=\{x: \exists y(x R y \in X)\} .
$$

Then $\bigcirc$ is interpreted by $|\bigcirc \varphi|=\langle R\rangle|\varphi|$, in accordance with (1.2). In order for $|\bigcirc \varphi|$ to be a proposition, a condition on $\triangleright$ and $R$ is imposed to ensure that $\langle R\rangle X$ is localised whenever $X$ is localised. This condition is called Modal Localisation (cf. Section 4). Further simple conditions on $R$ enforce $\langle R\rangle$ to be a nucleus on Prop, ensuring that the $\bigcirc$-axioms for a lax modality are valid in the model.

It is noteworthy that the function $X \mapsto j X$ is itself a nucleus on the lattice of up-sets, with Prop being the lattice of fixed points of $j$. Thus our models carry two nuclei, one to provide the semantics of the lax modality, and the other to provide the intuitionistic structure of the lattice of propositions.

In the following pages, the formal details of this theory are developed. The completeness theorem for QLL with respect to its cover semantics is shown by taking the MacNeille completion of the Lindenbaum algebra of the logic, and then converting this completion into a structural cover-system model of the kind we have been describing. On the way it is shown that any nucleus on a Heyting algebra can be extended to a nucleus on its MacNeille completion, and that any complete Heyting algebra is isomorphic to the lattice Prop of all propositions of a "localic" cover system. The methodology is also worked through for the quantified extensions of the systems CK (Constructive K) of [36] and CS4 (Constructive S4) of [3]. In addition, we give a cover semantics for the first-order intuitionistic modal logic studied in [45], which includes the axiom $\neg \diamond \perp$. At the end we also present an alternative completeness proof for (nonmodal) first-order intuitionistic logic itself with respect to the cover semantics, using a simple and explicit Henkin-style model construction whose points are the principal theories of the logic. 
It is important to be aware that our cover systems allow points that are covered by the empty set, and our models have

$$
x \models \perp \quad \text { iff } \quad x \triangleleft \emptyset .
$$

Points that satisfy $\perp$ are called fallible in $[13,14]$, and the PLL-models of those papers, as well as those of [36] and [3], include an arbitrary set $F$ of "fallible worlds", which serves as the truth set $|\perp|$. Models are required to have $F \subseteq|\varphi|$ in general, so that fallible worlds satisfy all sentences, ensuring the truth of $\perp \rightarrow \varphi$. Fallible worlds may be thought of as representing contexts of inconsistent requirements. PLL-models also allow points $x$ satisfying $\bigcirc \perp$, implying that there is some $y$ with $x R y \in F$. Intuitively, $y$ is a state that is reachable from $x$ under some constraint, but that constraint is inconsistent with $x$ [14, p. 8].

In the theory developed here, the notion of fallible world is represented by the set $j \emptyset=\{x: x \triangleleft \emptyset\}$ of points with the empty cover. This is built into the underlying structure of a cover system, rather being specified in various models on the system as their interpretation of $\perp$. Models of $\neg \diamond \perp$ have $\diamond \perp$ also satisfied at exactly the fallible (i.e. empty-covered) worlds. All of the logics we discuss, including non-modal first-order intuitionistic logic, will be shown to be characterised by models in which $j \emptyset$ is a singleton, i.e. there is exactly one fallible world.

In the light of this introduction and overview, we return to the question of the nature of $\bigcirc$ as a modality. Relative to Kripke's semantics it has two natural modellings, the box-like (1.1) and the partially diamond-like (1.5). Relative to cover semantics it has the diamond-like modelling (1.2). So is it a box or a diamond?

The moral of the story is that $\bigcirc$ need not be thought of as either. There is more to intuitionistic modal logic than will be found by generalising the behaviour of Boolean $\square$ and $\diamond$. It includes other phenomena that lose their significance when the law of excluded middle is present. ${ }^{4}$ Nuclei on Boolean algebras are uninteresting, ${ }^{5}$ as is the notion of a lax modality in Boolean logic. But in the intuitionistic setting this is a rich notion with a wide range of significant manifestations and applications.

Similarly, although the system CS4 has deductive postulates for $\square$ that are just like those of the Boolean box modality in the classical logic S4 (see Figure 2), here we interpreted the $\square$ of CS4 algebraically as a multiplicative interior operator, just as for classical S4, but model-theoretically as in (1.2) for a Boolean diamond (see (7.1)). CS4 has an apparently dual pair $\square$, $\diamond$ of modalities that can both be given the "existential" interpretation of (1.2). Likewise for CK. This suggests that there is a place for further clarification of just what "dual pair" might mean for non-Boolean modalities.

\section{Nuclei Under MacNeille Completion}

We begin by reviewing some possible properties of order-preserving functions.

Let $(H, \sqsubseteq)$ be a partially ordered set, and $f: H \rightarrow H$ a monotone (order-preserving) function, i.e. $a \sqsubseteq b$ implies $f a \sqsubseteq f b$. Then $f$ is

- idempotent if $f(f a)=f a$, for all $a \in H$;

- inflationary if $a \sqsubseteq f a$, for all $a \in H$;

- deflationary if $f a \sqsubseteq a$, for all $a \in H$;

- a closure operator if it is inflationary and idempotent;

- an interior operator if it is deflationary and idempotent.

If $\mathbf{H}$ is a lattice under $\sqsubseteq$ with binary meets $a \sqcap b$ and joins $a \sqcup b$, then $f$ is

- multiplicative if $f a \sqcap f b=f(a \sqcap b)$ for all $a, b \in H$;

\footnotetext{
${ }^{4}$ Further examples of this include the proof-intuitionistic modal logic of Kuznetsov and Muravitsky [30], which is related to the provability interpretation of modality, and its sublogic studied by Esakia [11], in which the modal connective is interpreted by the dual of the topological derivative operation. These logics have a box-like modality $\Delta$ with $\varphi \rightarrow \Delta \varphi$ as an axiom.

${ }^{5}$ Each element $a$ of a Heyting algebra defines the nucleus $f(x)=a \sqcup x$. On a Boolean algebra these are all the nuclei there are.
} 
- additive if $f a \sqcup f b=f(a \sqcup b)$ for all $a, b \in H$;

- a nucleus if it is a multiplicative closure operator.

Lemma 2.1 A monotone function is a nucleus iff it is inflationary and satisfies the inequalities

$$
\text { (i) } f(f a) \sqsubseteq f a, \quad \text { and }(i i) a \sqcap f b \sqsubseteq f(a \sqcap b) .
$$

Proof. A nucleus has $a \sqcap f b \sqsubseteq f a \sqcap f b \sqsubseteq f(a \sqcap b)$, by the inflationary, monotone and multiplicative properties.

Conversely, let monotone $f$ have the properties stated in the Lemma. Then $f a \sqsubseteq f(f a)$ by inflationarity, so $f$ is idempotent by (i). Also $f(a \sqcap b) \sqsubseteq f a \sqcap f b$ by monotonicity. But using (ii) twice and then (i),

$$
f a \sqcap f b \sqsubseteq f(f a \sqcap b) \sqsubseteq f(f(a \sqcap b)) \sqsubseteq f(a \sqcap b),
$$

so $f$ is multiplicative.

Recall that a Heyting algebra is a lattice $\mathbf{H}=(H, \sqsubseteq, \sqcap, \sqcup, 0)$ with least element 0 and an "implication" operation $a \Rightarrow b$ satisfying

$$
c \sqsubseteq a \Rightarrow b \quad \text { iff } \quad c \sqcap a \sqsubseteq b .
$$

The operation $\Rightarrow$ is monotone in its second argument and antitone (order-reversing) in its first: if $c \sqsubseteq a$ and $b \sqsubseteq d$, then $a \Rightarrow b \sqsubseteq c \Rightarrow d$. $\mathbf{H}$ has a greatest element 1 , with $1=a \Rightarrow a$ for any $a$.

Property (2.1) implies that the lattice is distributive. In fact if it is a complete lattice, i.e. every subset $\mathcal{X}$ of $H$ has a join $\bigsqcup \mathcal{X}$ and a meet $\Pi \mathcal{X}$, then (2.1) can be used to show that finite meets $\sqcap$ distribute over arbitrary joins $\bigsqcup$, meaning that

$$
a \sqcap(\bigsqcup \mathcal{X})=\bigsqcup\{a \sqcap b: b \in \mathcal{X}\}
$$

Conversely, any complete lattice satisfying (2.2) is a Heyting algebra in which

$$
a \Rightarrow b=\bigsqcup\{c: c \sqcap a \sqsubseteq b\} .
$$

Thus a complete Heyting algebra can be defined as a complete lattice satisfying (2.2). A complete Heyting algebra is also called a locale.

A completion of a lattice $\mathbf{H}$ is any complete lattice that has $\mathbf{H}$ as a sublattice. Each lattice $\mathbf{H}$ has a special completion $\overline{\mathbf{H}}$, known as the MacNeille completion, with the property that each member $x$ of $\overline{\mathbf{H}}$ satisfies

$$
x=\bigsqcup\{a \in H: a \sqsubseteq x\}=\prod\{b \in H: x \sqsubseteq b\},
$$

so is both a join and a meet of elements of $H$. In fact this property characterises $\overline{\mathbf{H}}$ uniquely up to isomorphism, and we will work with it in terms of this abstract characterisation. A crucial feature, which we make essential use of, is that the inclusion of $\mathbf{H}$ into $\overline{\mathbf{H}}$ preserves any existing joins and meets, i.e. if a subset of $H$ happens to have a join or meet in $\mathbf{H}$, then this is identical to its join or meet in $\overline{\mathbf{H}}$. For a distributive lattice $\mathbf{H}$, this is in stark contrast to the Stone completion [40], whose elements are certain sets of prime filters of $\mathbf{H}$. All completions preserve finite joins, but the Stone completion actually destroys every infinite join.

MacNeille's original construction [35] was a generalisation to posets of Dedkind's completion of the rationals by cuts. A detailed contemporary account of it may be found in [10, pp. 40-44]. The paper [41] analyses the lifting of functions from a lattice to its MacNeille completion, and we adopt some of its notation and terminology.

Any monotone function $f$ on a lattice $\mathbf{H}$ has two natural extensions to monotone functions $f^{\circ}$ and $f^{\bullet}$ on $\overline{\mathbf{H}}$, defined by

$$
\begin{aligned}
& f^{\circ} x=\bigsqcup\{f a: x \sqsupseteq a \in H\}, \\
& f^{\bullet} x=\prod\{f b: x \sqsubseteq b \in H\} .
\end{aligned}
$$

$f^{\circ}$ is the lower extension of $f$, and $f^{\bullet}$ is the upper extension (note that $f^{\circ} x \sqsubseteq f^{\bullet} x$ ). These are genuine extensions of $f$, i.e. they are identical to $f$ on $\mathbf{H}$.

It is readily checked that the upper extension preserves inflationarity and the inequality $f(f a) \sqsubseteq f a$, while the lower extension preserves deflationarity and the inequality $f a \sqsubseteq f(f a)$. Consequently: 
Theorem 2.2 The upper extension of a closure operator on a lattice is a closure operator on the MacNeille completion, while the lower extension of an interior operator is also an interior operator.

The class of Heyting algebras is closed under MacNeille completion: if $\mathbf{H}$ is a Heyting algebra, then its implication operation $\Rightarrow$ can be extended to $\overline{\mathbf{H}}$ by defining

$$
x \Rightarrow y=\prod\{a \Rightarrow b: x \sqsupseteq a \in H \text { and } y \sqsubseteq b \in H\} .
$$

This makes $\overline{\mathbf{H}}$ a Heyting algebra having $\mathbf{H}$ as a subalgebra. Although neither type of extension preserves multiplicativity of a function on a general lattice [41, $3.8 \& 3.10]$, for Heyting algebras we do have

Theorem 2.3 If $f$ is multiplicative on a Heyting algebra $\mathbf{H}$, then $f^{\circ}$ is multiplicative on $\overline{\mathbf{H}}$.

Proof. ${ }^{6}$ Let $x, y$ belong to $\overline{\mathbf{H}}$. In what follows, $a, b, c$ range over members of $\mathbf{H}$. By a double application of the $\sqcap$ - $\bigsqcup$-distribution law (2.2), and the multiplicativity of $f$, we reason that

$$
f^{\circ} x \sqcap f^{\circ} y=\left(\bigsqcup_{a \sqsubseteq x} f a\right) \sqcap\left(\bigsqcup_{b \sqsubseteq y} f b\right)=\bigsqcup_{a \sqsubseteq x, b \sqsubseteq y}(f a \sqcap f b) \sqsubseteq \bigsqcup_{a \sqsubseteq x, b \sqsubseteq y} f(a \sqcap b) \sqsubseteq \bigsqcup_{c \sqsubseteq x \sqcap y} f c=f^{\circ}(x \sqcap y) .
$$

But $f^{\circ}(x \sqcap y) \sqsubseteq f^{\circ} x \sqcap f^{\circ} y$ by monotonicity.

Corollary 2.4 The lower extension of a multiplicative interior operator on a Heyting algebra is also a multiplicative interior operator.

Proof. $\quad$ By Theorems 2.2 and 2.3.

We will now show that the upper extension of a nucleus is a nucleus. This requires a new analysis. We prove a more general result that will also be applied later to algebraic models of the intuitionistic modal logic CS4 (see Theorem 7.3).

Theorem 2.5 Let $f$ and $g$ be monotone functions on a Heyting algebra $\mathbf{H}$ such that $f a \sqcap g b \sqsubseteq g(a \sqcap b)$ for all $a, b \in \mathbf{H}$. Then $f^{\circ} x \sqcap g^{\bullet} y \sqsubseteq g^{\bullet}(x \sqcap y)$ for all $x, y \in \overline{\mathbf{H}}$.

Proof. $\quad$ Let $x, y \in \overline{\mathbf{H}}$. Take any $a, b \in \mathbf{H}$ with $a \sqsubseteq x$ and $x \sqcap y \sqsubseteq b$. Then $y \sqsubseteq x \Rightarrow b \sqsubseteq a \Rightarrow b$, so

$$
f a \sqcap g^{\bullet} y \sqsubseteq f a \sqcap g^{\bullet}(a \Rightarrow b)=f a \sqcap g(a \Rightarrow b)
$$

(as $a \Rightarrow b \in \mathbf{H})$. But $f a \sqcap g(a \Rightarrow b) \sqsubseteq g(a \sqcap(a \Rightarrow b))$ by the assumption on $f$ and $g$, and $g(a \sqcap(a \Rightarrow b)) \sqsubseteq g b$ as $a \sqcap(a \Rightarrow b) \sqsubseteq b$, so altogether we get $f a \sqcap g^{\bullet} y \sqsubseteq g b$.

Applying this to a particular $b$, we reason that

$$
f^{\circ} x \sqcap g^{\bullet} y=\left(\bigsqcup_{a \sqsubseteq x} f a\right) \sqcap g^{\bullet} y=\bigsqcup_{a \sqsubseteq x}\left(f a \sqcap g^{\bullet} y\right) \sqsubseteq g b .
$$

Hence $f^{\circ} x \sqcap g^{\bullet} y \sqsubseteq \prod_{x \sqcap y \sqsubseteq b} g b=g^{\bullet}(x \sqcap y)$.

Corollary 2.6 The upper extension of a nucleus on a Heyting algebra is also a nucleus.

Proof. Let $g$ be a nucleus on Heyting algebra $\mathbf{H}$. Then by Theorem $2.2, g^{\bullet}$ is a closure operator on $\overline{\mathbf{H}}$, i.e. inflationary and idempotent.

Now by Lemma 2.1, $a \sqcap g b \sqsubseteq g(a \sqcap b)$. Let $f$ be the identity function on $\mathbf{H}$. Then $f^{\circ}$ is the identity on $\overline{\mathbf{H}}$, so by Theorem 2.5, $x \sqcap g^{\bullet} y \sqsubseteq g^{\bullet}(x \sqcap y)$. Hence by Lemma 2.1 again, $g^{\bullet}$ is a nucleus.

The observation that upper MacNeille extensions preserve nuclei appears to be new. It would be of interest to place it, and Theorem 2.5, within the context of general studies of properties preserved by MacNeille completion, like those of $[19,41]$.

\footnotetext{
${ }^{6} \mathrm{~A}$ generalisation of this was shown in [22, p. 222] with $\overline{\mathbf{H}}$ a quantale. For $\overline{\mathbf{H}}$ a locale, the result was extended to functions of more than one argument in [41, 3.16].
} 


\section{Localic Cover Systems}

Let $\mathcal{S}=(S, \leq, \triangleleft)$, be a structure comprising a partial order $\leq$ on the set $S$, and a binary relation $\triangleleft$ from $S$ to its powerset $\mathcal{P} S$. When $x \triangleleft C$, where $x \in S$ and $C \subseteq S$, we say that $x$ is covered by $C$, and write this also as $C \triangleright x$, saying that $C$ covers $x$ or that $C$ is an $x$-cover. We sometimes write $y \geq x$ when $x \leq y$, and say that $y$ refines $x$.

As a possible aid to the intuition, think of $S$ as a set of states, computational or other, that each have a certain information content. View refinement as a relation of information increase, so that if $y \geq x$, then the information content of $y$ includes that of $x$. Interpret covering as a relation expressing commonality of information content, so that if $C \triangleright x$ then the information content of $x$ consists of that which is common to all the states in $C$. At the end of the paper there is a model construction that supports this interpretation. For the standard topological construction of cover systems, see Example 3.1 below.

For each subset $X$ of $S$, define

$$
j X=\{x \in S: \exists C(x \triangleleft C \subseteq X)\} .
$$

Thus a member $x$ of $j X$ is a local member of $X$, in the sense that it is covered by a set $C$ of members of $X$. $X$ is called localised if $j X \subseteq X$. A localised set is one for which membership of it is a local property.

An up-set is a subset $X$ of $S$ that is closed upward under the partial order: $y \geq x \in X$ implies $y \in X$. For an arbitrary $X \subseteq S$,

$$
\uparrow X=\{y \in S:(\exists x \in X) x \leq y\}
$$

is the smallest up-set including $X$. It consists of those points that refine some member of $X$. Thus $X$ is an up-set iff $\uparrow X=X$. For $x \in S$,

$$
\uparrow x=\uparrow\{x\}=\{y: x \leq y\}
$$

is the smallest up-set containing $x$, and will be called the cone at $x$.

The collection $U p(\mathcal{S})$ of all up-sets of $\mathcal{S}$ is a locale under the partial order $\subseteq$ of set inclusion, with the join $\sqcup \mathcal{X}$ and meet $\sqcap \mathcal{X}$ of any collection $\mathcal{X}$ of up-sets being the set union $\bigcup \mathcal{X}$ and intersection $\bigcap \mathcal{X}$ respectively, while $0=\emptyset$ and $1=S$. In this case, the infinite distributive law (2.2) evidently holds, and the Heyting implication is given by

$$
X \Rightarrow Y=\{x \in S: \uparrow x \cap X \subseteq Y\}
$$

for any up-sets $X, Y$. Thus $x \in X \Rightarrow Y$ iff $x \leq y \in X$ implies $y \in Y$, so $X \Rightarrow Y$ embodies the semantics for intuitionistic implication of [29].

A subset $Y$ refines a subset $X$ if $Y \subseteq \uparrow X$, i.e. every member of $Y$ refines some member of $X$. We call $\mathcal{S}$ a cover system if it satisfies the following axioms, for all $x \in S$ :

- Existence: there exists an $x$-cover $C \subseteq \uparrow x$;

- Transitivity: if $x \triangleleft C$ and for all $y \in C, y \triangleleft C_{y}$, then $x \triangleleft \bigcup_{y \in C} C_{y}$.

- Refinement: if $x \leq y$, then every $x$-cover can be refined to a $y$-cover, i.e. if $C \triangleright x$, then there exists a $C^{\prime} \triangleright y$ with $C^{\prime} \subseteq \uparrow C$.

A localised up-set will be called a proposition. $\operatorname{Prop}(\mathcal{S})$ is the collection of all propositions of $\mathcal{S}$.

\section{Example 3.1}

Let $S$ be the set of open subsets of some topological space, with $x \leq y$ iff $x \supseteq y$ and $C \triangleright x$ iff $\bigcup C=x$. Then $\triangleright$ captures the usual notion of open cover, and " $Y$ refines $X$ " has its usual meaning for open covers that every member of $Y$ is included in a member of $X$ [38, p. 245]. For the Refinement axiom, observe that if $\bigcup C=x \supseteq y$, then $\bigcup C^{\prime}=y$ where $C^{\prime}=\{y \cap c: c \in C\} \subseteq \uparrow C$.

In this cover system, each cone $\uparrow x$ is a proposition, but a union $\uparrow x \cup \uparrow y$ of cones need not be localised. If $z$ is any subset of the ambient space, $\{x \in S: z \cap x=\emptyset\}$ is a proposition.

\section{Example 3.2}

Let $L$ be any complete lattice, with partial order $\sqsubseteq$ and infinite join operation $\bigsqcup$. Then a cover system on $L$ is defined by putting $x \leq y$ iff $y \sqsubseteq x$ and $C \triangleright x$ iff $x \sqsubseteq \bigsqcup C$. In this case the propositions are exactly the cones [22, Theorem 6]: if $X$ is a localised up-set, put $x=\bigsqcup X$ to get $x \triangleleft X$, hence $x \in X$, and so $X=\uparrow x$. 
Lemma 3.3 In any cover system $\mathcal{S}$, if $X$ is an up-set then so is $j X$. The function $j: U p(\mathcal{S}) \rightarrow U p(\mathcal{S})$ is a closure operator on the locale of up-sets of $\mathcal{S}$.

Proof. If $X$ is an up-set and $y \geq x \in j X$, then there exists $C$ with $x \triangleleft C \subseteq X$, so by the Refinement axiom there exists $C^{\prime}$ with $y \triangleleft C^{\prime} \subseteq \uparrow C \subseteq \uparrow X=X$, hence $y \in j X$. Thus $j X$ is an up-set.

It is straightforward that $X \subseteq Y$ implies $j X \subseteq j Y$, so $j$ is monotone.

For the inflationary property, if $x \in X$ with $X$ an up-set, then by the Existence axiom there exists $C$ with $x \triangleleft C \subseteq \uparrow x \subseteq X$, so $x \in j X$. Thus $X \subseteq j X$ for all $X \in U p(\mathcal{S})$. For idempotence, first observe that if $X$ is an up-set, $j X \subseteq j(j X)$ follows by inflationarity, since $j X$ is also an up-set. But the converse inclusion $j(j X) \subseteq j X$ holds for any $X$ by Transitivity. For, if there is an $x$-cover $C \subseteq j X$, then for all $y \in C$ there is a $y$-cover $C_{y} \subseteq X$, and so $\bigcup_{y \in C} C_{y}$ is an $x$-cover included in $X$, showing $x \in j X$.

This Lemma implies that if $X$ is a proposition (i.e. if $j X \subseteq X=\uparrow X$ ), then $j X=X$ by inflationarity. In general, $X$ is a proposition iff $X=\uparrow X=j X . j \uparrow X$ is the smallest proposition including an arbitrary $X$, and $j \uparrow x$ is the smallest proposition containing the element $x$. The smallest proposition including an up-set $X$ is just $j X$, so in fact $j: U p(\mathcal{S}) \rightarrow \operatorname{Prop}(\mathcal{S})$.

Now the set $\operatorname{Prop}(\mathcal{S})$ of propositions of a cover system is a complete lattice (see below), but it need not be a locale, or even be finitely distributive - see Example 3.2. To see what is required for $\operatorname{Prop}(\mathcal{S})$ to be a locale, consider that in the topological case of Example 3.1, $x \triangleleft C$ was defined to mean that $x=\bigcup C$. Now the intuitive idea of open covering is sometimes formulated by requiring only that $x \subseteq \bigcup C$, but this violates the condition $C \subseteq \uparrow x$ which holds when $x=\bigcup C$. On the other hand, if $x \subseteq \bigcup C$, then $C$ can be refined to $C^{\prime}=\{x \cap c: c \in C\}$, which does have $x \triangleleft C^{\prime} \subseteq \uparrow x$.

With this in mind, a cover system will be called localic if it satisfies:

every $x$-cover can be refined to an $x$-cover that is included in $\uparrow x$.

Lemma 3.4 In a localic cover system $\mathcal{S}$ :

(1) $j$ is a nucleus on $U p(\mathcal{S})$.

(2) If $X$ is an up-set and $Y$ is a proposition, then $X \Rightarrow Y$ is a proposition, where $X \Rightarrow Y$ is as in (3.1).

Proof.

(1) In view of Lemmas 3.3 and 2.1, it remains only to show $X \cap j Y \subseteq j(X \cap Y)$ for up-sets $X, Y$. So let $x \in X \cap j Y$. Then there is an $x$-cover $C \subseteq Y$. Hence by the localic condition, there is an $x$-cover $C^{\prime} \subseteq \uparrow C$ with $C^{\prime} \subseteq \uparrow x$. But $\uparrow x \subseteq X$ as $X$ is an up-set, and $\uparrow C \subseteq Y$ as $Y$ is an up-set. So this implies $x \triangleleft C^{\prime} \subseteq X \cap Y$, showing that $x \in j(X \cap Y)$.

(2) Let $X \in U p(\mathcal{S})$ and $Y \in \operatorname{Prop}(\mathcal{S})$. Then $X \Rightarrow Y$ is an up-set. To show it is localised, observe that since $j$ is a nucleus,

$$
X \cap j(X \Rightarrow Y) \subseteq j(X \cap(X \Rightarrow Y)) \subseteq j Y .
$$

But $j Y=Y$ as $Y$ is a proposition, so $X \cap j(X \Rightarrow Y) \subseteq Y$, hence $j(X \Rightarrow Y) \subseteq(X \Rightarrow Y)$ as required.

Theorem 3.5 In a localic cover system, the set of propositions forms a locale under the inclusion order $\subseteq$, in which

$$
\begin{aligned}
\sqcap \mathcal{X} & =\bigcap \mathcal{X} \\
\bigsqcup \mathcal{X} & =j(\bigcup \mathcal{X}) \\
X \Rightarrow Y & =\{x \in S: \uparrow x \cap X \subseteq Y\} \\
0 & =j \emptyset=\{x: \emptyset \triangleright x\} .
\end{aligned}
$$

Proof. The propositions are just the up-sets that are fixed by $j$, i.e.

$$
\operatorname{Prop}(\mathcal{S})=\{X \in U p(\mathcal{S}): j X=X\} .
$$

It is a general fact about a closure operator on a complete lattice that the set of fixed points is a complete lattice under the same meet operation, with joins being the closures of the joins in the original lattice. ${ }^{7}$

\footnotetext{
${ }^{7}$ The operation $X \mapsto \uparrow X$ is itself a closure operator on the powerset of $S$, whose lattice of fixed points is $U p(\mathcal{S})$.
} 
More directly, it is readily seen that if $\mathcal{X} \subseteq \operatorname{Prop}(\mathcal{S})$, then $\bigcap \mathcal{X}$ is a proposition, and hence is the meet $\Pi \mathcal{X}$ in $\operatorname{Prop}(\mathcal{S})$; and $j(\bigcup \mathcal{X})$ is the least proposition including the up-set $\bigcup \mathcal{X}$, hence is the join $\bigsqcup \mathcal{X}$ in $\operatorname{Prop}(\mathcal{S})$.

For $\operatorname{Prop}(\mathcal{S})$ to be a locale, it must have an operation $\Rightarrow$ satisfying (2.1). Since we want (3.1) to hold, we define $X \Rightarrow Y$ by the equation (3.1), and conclude from Lemma 3.4 that Prop $(\mathcal{S})$ is closed under this operation. But this operation has

$$
X \cap Z \subseteq Y \quad \text { iff } \quad Z \subseteq X \Rightarrow Y
$$

for all up-sets $X, Y, Z$, so in particular satisfies this for all $X, Y, Z \in \operatorname{Prop}(\mathcal{S})$, giving the desired result.

Since $\emptyset$ is an up-set, $j \emptyset$ is the least proposition including $\emptyset$, hence the least element of $\operatorname{Prop}(\mathcal{S})$. Note that it may be that $j \emptyset \neq \emptyset$, as we will see after the next Theorem.

An obvious strengthening of the localic condition is the requirement that every $x$-cover be included in $\uparrow x$, i.e. $C \triangleright x$ implies $C \subseteq \uparrow x$, as in Example 3.1. This condition, which we shall call strictly localic, is intrinsically built into generalisations of topological open-cover systems to "Grothendieck topologies" and other abstract categorical settings $[27,32,6] .^{8}$ To define cover semantics for non-distributive logics, the localic condition has to be abandoned, as in [22].

Theorem 3.6 Every locale is isomorphic to the locale of all propositions of some strictly localic cover system.

Proof. $\quad$ Let $\mathbf{H}=(H, \sqsubseteq, \sqcap, \sqcup, \Rightarrow, 0)$ be a locale. Define $\mathcal{S}_{\mathbf{H}}=(S, \leq, \triangleleft)$ by putting $S=H, x \leq y$ iff $y \sqsubseteq x$, and $x \triangleleft C$ iff $x=\bigsqcup C$ in $\mathbf{H}$ (cf. Examples 3.1 and 3.2). Then the up-set $\uparrow x$ of $x$ under $\leq$ is just the down-set $(x]=\{y: y \sqsubseteq x\}$ of $x$ under $\sqsubseteq$. We verify the cover system axioms for $\mathcal{S}_{\mathbf{H}}$ :

- Existence: putting either $C=\{x\}$ or $C=(x]$ will do to provide a $C \subseteq(x]$ with $x=\bigsqcup C$, hence $x \triangleleft C \subseteq \uparrow x$.

- Transitivity: if $x=\bigsqcup C$, and for all $y \in C, y=\bigsqcup C_{y}$, then $x=\bigsqcup_{y \in C}\left(\bigsqcup C_{y}\right)=\bigsqcup\left(\bigcup_{y \in C} C_{y}\right)$.

- Refinement: if $x \leq y$ and $C$ is an $x$-cover, let $C^{\prime}=\{y \sqcap c: c \in C\}$. Then $y=y \sqcap x=y \sqcap \bigsqcup C=\bigsqcup C^{\prime}$. Hence $C^{\prime}$ is a $y$-cover, and $C^{\prime}$ refines $C$ as $c \leq y \sqcap c$.

Since $x=\bigsqcup C$ implies $C \subseteq(x]=\uparrow x, \mathcal{S}_{\mathbf{H}}$ is strictly localic is the sense mentioned above. Now notice that every cone $(x]$ is localised, hence is a proposition of $\mathcal{S}$, for if $y \triangleleft C \subseteq(x]$, then $y=\bigsqcup C \sqsubseteq x$, so $y \in(x]$. But if $X$ is any proposition, and we put $x=\bigsqcup X$, then $x \triangleleft X$, so $x \in X$ as $X$ is localised, hence $X=(x]$ as $X$ is a down-set under $\sqsubseteq$.

Thus the map $x \mapsto(x]$ takes $H$ onto the set of propositions of $\mathcal{S}_{\mathbf{H}}$. The map is order-invariant: $x \sqsubseteq y$ iff $(x] \subseteq(y]$. From this it follows that it is an isomorphism between the ordered sets $(H$, $)$ and $\left(\operatorname{Prop}\left(\mathcal{S}_{\mathbf{H}}\right), \subseteq\right)$. But the operations $\sqcap, \bigsqcup, \Rightarrow, 0$ are all characterised by the order structure (cf. (2.3)), so $x \mapsto(x]$ is an isomorphism between $\mathbf{H}$ and $\operatorname{Prop}\left(\mathcal{S}_{\mathbf{H}}\right)$ as complete Heyting algebras.

Note that in the system $\mathcal{S}_{\mathbf{H}}$ of this proof, we have $0 \triangleleft \emptyset$, where 0 is the least element of $\mathbf{H}$, and in fact $j \emptyset=\{0\}$, since $x \triangleleft \emptyset$ iff $x=\bigsqcup \emptyset=0$. Thus in locales of the form $\operatorname{Prop}\left(\mathcal{S}_{\mathbf{H}}\right)$, the least proposition is non-empty.

Corollary 3.7 Every Heyting algebra $\mathbf{H}$ can be embedded into the locale of all propositions of some strictly localic cover system, by an injective homomorphism that preserves all existing joins and meets.

Proof. Compose the inclusion $\mathbf{H} \hookrightarrow \overline{\mathbf{H}}$ of $\mathbf{H}$ into its MacNeille completion with the isomorphism $\overline{\mathbf{H}} \rightarrow \operatorname{Prop}\left(\mathcal{S}_{\overline{\mathbf{H}}}\right)$ given by Theorem 3.6.

${ }^{8}$ Those settings typically use down-sets rather than up-sets. Here we are following the order-convention most commonly used in the relational semantics of intuitionistic, relevant and other substructural logics. 


\section{Modal Operators}

We will take a modal operator to be any monotone function on a poset. A modal Heyting algebra $(\mathbf{H}, m)$ consists of a monotonic function $m$ on a Heyting algebra $\mathbf{H}$. This is a modal locale if $\mathbf{H}$ is a locale.

To construct such algebras, let $R$ be a binary relation on the underlying set of a cover system $\mathcal{S}=$ $(S, \leq, \triangleleft)$. Then a modal operator $\langle R\rangle$ on $(\mathcal{P}(S), \subseteq)$ is defined by

$$
\langle R\rangle X=\{x \in S: \exists y(x R y \in X)\} .
$$

$(\mathcal{S}, R)$ is a modal cover system if it satisfies

- Confluence: if $x \leq y$ and $x R z$, then there exists $w$ with $z \leq w$ and $y R w$;

- Modal Localisation: if there exists an $x$-cover included in $\langle R\rangle X$, then there exists a $y$ with $x R y$ and a $y$-cover included in $X$.

Confluence ensures that $\langle R\rangle X$ is an up-set whenever $X$ is an up-set, so that $\langle R\rangle$ becomes a modal operator on $(U p(\mathcal{S}), \subseteq)$. Modal Localisation states that if $x \in j(\langle R\rangle X)$, then there is a $y \in j X$ with $x R y$; so that if $j X \subseteq X$ then $x \in\langle R\rangle X$. This ensures that if $X$ is localised, then so is $\langle R\rangle X$.

Thus in a modal cover system,

$$
\langle R\rangle X \text { is a proposition whenever } X \text { is a proposition, }
$$

so $\langle R\rangle$ becomes a modal operator on $(\operatorname{Prop}(\mathcal{S}), \subseteq)$. Hence if $(\mathcal{S}, R)$ is a localic modal cover system, then the algebra $(\operatorname{Prop}(\mathcal{S}),\langle R\rangle)$ is a modal locale. ${ }^{9}$

$(\mathcal{S}, R)$ will be called nuclear if $R$ is a preorder, i.e. reflexive and transitive, such that

$$
x R y \text { implies } \exists z \geq y(x \leq z \text { and } x R z) .
$$

This last condition is a weakening of the requirement, used in the models of lax logic of [14], that $R$ be a subrelation of $\leq$. For if $x R y$ implies $x \leq y$, then (4.2) holds by putting $z=y$.

Lemma 4.1 If a modal cover system $(\mathcal{S}, R)$ is nuclear, then the modal operator $\langle R\rangle$ is a nucleus on $U p(\mathcal{S})$, and hence a nucleus on $\operatorname{Prop}(\mathcal{S})$.

Proof. It is well known that if $R$ is reflexive then $X \subseteq\langle R\rangle X$, and if $R$ is transitive then $\langle R\rangle\langle R\rangle X \subseteq$ $\langle R\rangle X$; so if $R$ is a pre-order then $\langle R\rangle$ is a closure operator on $\mathcal{P}(S)$, and therefore a closure operator on $U p(\mathcal{S})$, and on $\operatorname{Prop}(\mathcal{S})$.

The extra condition (4.2) ensures that $X \cap\langle R\rangle Y \subseteq\langle R\rangle(X \cap Y)$ whenever $X$ and $Y$ are up-sets, from which by Lemma 2.1 it follows that $\langle R\rangle$ is a nucleus on $U p(\mathcal{S})$, hence on $\operatorname{Prop}(\mathcal{S})$. For the details: if $x \in X \cap\langle R\rangle Y$, then $x \in X$ and $x R y$ for some $y \in Y$, so taking a $z$ as given by (4.2) we get $z \in X$ as $x \leq z$ and $X$ is an up-set, and similarly $z \in Y$ as $z \leq y$, hence $x \in\langle R\rangle(X \cap Y)$ as $x R z \in X \cap Y$.

We have now seen that from any nuclear localic modal cover system $(\mathcal{S}, R)$ we can build a modal locale $(\operatorname{Prop}(\mathcal{S}),\langle R\rangle)$ whose modal operator $\langle R\rangle$ is a nucleus. To proceed in the converse direction, take any modal locale $(\mathbf{H}, m)$ and define a relation $R_{m}$ on the localic cover system $\mathcal{S}_{\mathbf{H}}$ constructed in the proof of Theorem 3.6 by putting $x R_{m} y$ iff $x \sqsubseteq m y$.

Theorem $4.2\left(\mathcal{S}_{\mathbf{H}}, R_{m}\right)$ is a localic modal cover system, and the map $x \mapsto(x]$ is an isomorphism from $(\mathbf{H}, m)$ onto the modal locale $\left(\operatorname{Prop}\left(\mathcal{S}_{\mathbf{H}}\right),\left\langle R_{m}\right\rangle\right)$. Moreover, if $m$ is a nucleus on $\mathbf{H}$, then $\left(\mathcal{S}_{\mathbf{H}}, R_{m}\right)$ is nuclear, and so $\left\langle R_{m}\right\rangle$ is a nucleus on $\operatorname{Prop}\left(\mathcal{S}_{\mathbf{H}}\right)$.

Proof. We already know from Theorem 3.6 that $\mathcal{S}_{\mathbf{H}}$ is a localic cover system. To show that $\left(\mathcal{S}_{\mathbf{H}}, R_{m}\right)$ is modal requires proving the Confluence and Modal Localisation properties.

Confluence: if $x \leq y$ and $x R_{m} z$, then $y \sqsubseteq x \sqsubseteq m z$. Hence Confluence holds in the stronger form that if $x \leq y$ and $x R_{m} z$, then $y R_{m} z$.

Modal Localisation: suppose there is a $C$ with $x \triangleleft C \subseteq\left\langle R_{m}\right\rangle X$. Then $x=\sqcup C$ in $\mathbf{H}$. Let $C^{\prime}=\left\{z \in X: \exists c \in C\left(c R_{m} z\right)\right\}$ and put $y=\bigsqcup C^{\prime}$. Then $C^{\prime}$ is a $y$-cover included in $X$, and so it remains only to show that $x R_{m} y$. But if $c \in C$, then $c \in\left\langle R_{m}\right\rangle X$ by supposition, so there exists $z$ with $c R_{m} z \in X$.

\footnotetext{
${ }^{9}$ This only requires Modal Localisation to hold when $X$ is a proposition.
} 
Then $c \sqsubseteq m z$ and $z \in C^{\prime}$, so $z \sqsubseteq y$, hence $m z \sqsubseteq m y$ as $m$ is monotone, and thus $c \sqsubseteq m y$. Therefore $x=\bigsqcup C \sqsubseteq m y$, giving $x R_{m} y$ as required. ${ }^{10}$

Now Theorem 3.6 showed that $x \mapsto(x]=\{y: y \sqsubseteq x\}$ is an isomorphism between the locales $\mathbf{H}$ and $\operatorname{Prop}\left(\mathcal{S}_{\mathbf{H}}\right)$, so here we need to show in addition that it preserves the modal operators $m$ and $\left\langle R_{m}\right\rangle$, i.e. that $(m x]=\left\langle R_{m}\right\rangle(x]$. But if $y \in(m x]$, then $y R_{m} x \in(x]$, showing that $y \in\left\langle R_{m}\right\rangle(x]$. Conversely, if $y \in\left\langle R_{m}\right\rangle(x]$, then $y R_{m} z \sqsubseteq x$ for some $z$, so $y \sqsubseteq m z \sqsubseteq m x$, implying $y \in(m x]$.

This completes the proof of the first sentence of the Theorem. Now suppose $m$ is a nucleus. Then $R_{m}$ is reflexive as $x \sqsubseteq m x$; and transitive because if $x \sqsubseteq m y$ and $y \sqsubseteq m z$, then $m y \sqsubseteq m m z \sqsubseteq m z$, giving $x \sqsubseteq m z$. To show $R_{m}$ satisfies (4.2), suppose $x R_{m} y$. Then $x \sqsubseteq x \sqcap m y \sqsubseteq m(x \sqcap y)$, so putting $z=x \sqcap y$ we have $x R_{m} z$, while $y \leq z$ and $x \leq z$ because $\leq$ is the inverse relation of $\sqsubseteq$.

This shows that $\left(\mathcal{S}_{\mathbf{H}}, R_{m}\right)$ is nuclear. Hence $\left\langle R_{m}\right\rangle$ is a nucleus by Lemma 4.1 .

\section{Quantified Lax Logic}

We adopt a standard syntax for first-order intuitionistic modal predicate logic. Let $\mathcal{L}$ be a signature, i.e. a collection of individual constants $c$, and predicate symbols $P$ with specified arities $n<\omega$. An $\mathcal{L}$-term $\tau$ is any individual constant $c \in \mathcal{L}$, or individual variable $v$ from some fixed denumerable list of such variables. An atomic $\mathcal{L}$-formula is any expression $P \tau_{1} \cdots \tau_{n}$ where $P \in \mathcal{L}$ is $n$-ary, and the $\tau_{i}$ are $\mathcal{L}$-terms. The set of all $\mathcal{L}$-formulas is generated in the usual way from the atomic $\mathcal{L}$-formulas by using the propositional connectives $\wedge$ (conjunction), $\vee$ (disjunction), and $\rightarrow$ (implication); a constant formula $\perp$; the quantifiers $\forall v, \exists v$ for all variables $v$; and a unary modality $\bigcirc$. The negation $\neg \varphi$ is defined to be the formula $\varphi \rightarrow \perp$, while the bimplication $\varphi \leftrightarrow \psi$ is defined to be $(\varphi \rightarrow \psi) \wedge(\psi \rightarrow \varphi)$.

Substitution of terms for free variables is symbolised by writing $\varphi(\tau / v)$ for the $\mathcal{L}$-formula obtained by substituting $\tau$ in place of all free occurrences of $v$ in $\varphi$, on condition that $\tau$ is freely substitutable for $v$ in $\varphi$. If this condition fails, then $\varphi(\tau / v)$ will be taken to be the result of substituting $\tau$ for free $v$ in some bound alphabetic variant of $\varphi$ in which $\tau$ is freely substitutable for $v$.

If $U$ is any set, let $\mathcal{L}^{U}$ be the extension of $\mathcal{L}$ to include the members of $U$ as individual constants.

A Hilbert-style axiomatisation of the logic QLL is presented in Figure 1. It consists of a standard axiomatisation of first-order intuitionistic predicate logic, together with three axioms for the modality $\bigcirc$. The first seven rows comprise an adequate set of axioms for intuitionistic propositional logic, one that is convenient for formulations in which $\perp$ is primitive and negation is defined [39, p30]. We write QLL $\vdash \varphi$, or just $\vdash \varphi$, to mean that the formula $\varphi$ is derivable as a theorem in the logic of Figure 1 .

The axioms for $\bigcirc$ correspond to the characterisation of a nucleus given in Lemma 2.1. There are many possible variations on the axiomatisation of a lax modality; for instance the third axiom could be replaced by $(\varphi \rightarrow \psi) \rightarrow(\bigcirc \varphi \rightarrow \bigcirc \psi)$, or all three axioms could be replaced by the single axiom $(\varphi \rightarrow \bigcirc \psi) \leftrightarrow(\bigcirc \varphi \rightarrow \bigcirc \psi)$ (see [34] and [20] for discussion).

A $Q L L$-model for $\mathcal{L}$ is a structure $\mathcal{M}=\left(\mathcal{S}, R, U,|\cdot|^{\mathcal{M}}\right)$, where $(\mathcal{S}, R)$ is a nuclear localic cover system, $U$ is a non-empty set (universe of individuals), and $|\cdot|^{\mathcal{M}}$ is an interpretation function assigning

- to each individual constant $c \in \mathcal{L}$ an element $|c|^{\mathcal{M}} \in U ;$ and

- to each $n$-ary predicate symbol $P \in \mathcal{L}$, a function $|P|^{\mathcal{M}}: U^{n} \rightarrow \operatorname{Prop}(\mathcal{S})$.

An $\mathcal{L}$-model automatically extends to an $\mathcal{L}^{U}$-model by putting $|c|^{\mathcal{M}}=c$ for all $c \in U$. In this way, the members of $U$ function as self-naming constants.

Intuitively, $|P|^{\mathcal{M}}\left(u_{1}, \ldots, u_{n}\right)$ is the proposition asserting that the predicate $P$ holds of the $n$-tuple of individuals $\left(u_{1}, \ldots, u_{n}\right)$.

A model $\mathcal{M}$ has a truth/satisfaction relation $\mathcal{M}, x \models \varphi$ between elements $x \in S$ and sentences $\varphi$ of $\mathcal{L}^{U}$, with associated truth-sets

$$
|\varphi|^{\mathcal{M}}=\{x \in S: \mathcal{M}, x \models \varphi\} .
$$

These notions are defined by induction on the length of $\varphi$, as follows.

\footnotetext{
${ }^{10}$ This holds for any subset $X$ of $\mathcal{S}_{\mathbf{H}}$, even though it is only required to hold for $X$ a proposition in order for $\left\langle R_{m}\right\rangle$ to be an operator on $\operatorname{Prop}\left(\mathcal{S}_{\mathbf{H}}\right)$.
} 


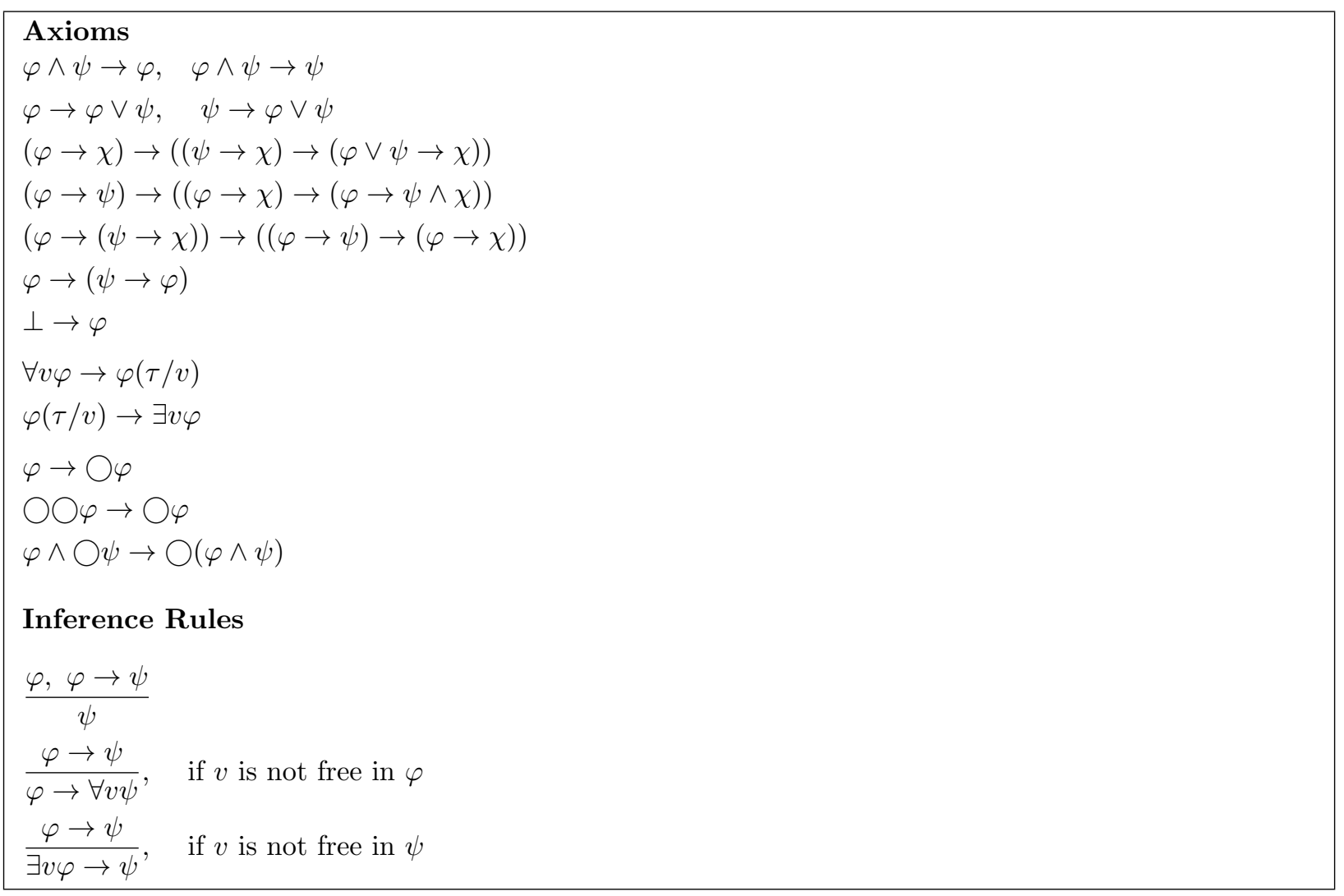

Figure 1: Axioms and Rules for Quantified Lax Logic

$$
\begin{array}{lll}
\mathcal{M}, x \models P c_{1} \cdots c_{n} & \text { iff } & x \in|P|^{\mathcal{M}}\left(\left|c_{1}\right|^{\mathcal{M}}, \ldots,\left|c_{n}\right|^{\mathcal{M}}\right) \\
\mathcal{M}, x \models \varphi \wedge \psi & \text { iff } & \mathcal{M}, x \mid=\varphi \text { and } \mathcal{M}, x \models \psi \\
\mathcal{M}, x \models \varphi \vee \psi & \text { iff } & \text { there is an } x \text {-cover } C \subseteq|\varphi|^{\mathcal{M}} \cup|\psi|^{\mathcal{M}} \\
\mathcal{M}, x \models \varphi \rightarrow \psi & \text { iff } & \text { for all } y \geq x, \mathcal{M}, y \models \varphi \text { implies } \mathcal{M}, y \models \psi \\
\mathcal{M}, x \models \perp & \text { iff } & \emptyset \triangleright x \\
\mathcal{M}, x \models \forall v \varphi & \text { iff } \quad \text { for all } c \in U, \mathcal{M}, x \models \varphi(c / v) \\
\mathcal{M}, x \models \exists v \varphi & \text { iff } \quad \text { there is an } x \text {-cover } C \subseteq \bigcup_{c \in U}|\varphi(c / v)|^{\mathcal{M}} \\
\mathcal{M}, x \models \bigcirc \varphi & \text { iff } & \text { for some } y, x R y \text { and } \mathcal{M}, y \models \varphi .
\end{array}
$$

These conditions can be expressed entirely in terms of the truth sets $|\varphi|^{\mathcal{M}}$, using the algebra of the set $\operatorname{Prop}(\mathcal{S})$ of propositions of $\mathcal{S}$, by

$$
\begin{array}{rlrl}
\left|P c_{1}, \ldots, c_{n}\right|^{\mathcal{M}} & =|P|^{\mathcal{M}}\left(\left|c_{1}\right|^{\mathcal{M}}, \ldots,\left|c_{n}\right|^{\mathcal{M}}\right) & \\
|\varphi \wedge \psi|^{\mathcal{M}} & =|\varphi|^{\mathcal{M}} \cap|\psi|^{\mathcal{M}} & & =|\varphi|^{\mathcal{M}} \sqcap|\psi|^{\mathcal{M}} \\
|\varphi \vee \psi|^{\mathcal{M}} & =j\left(|\varphi|^{\mathcal{M}} \cup|\psi|^{\mathcal{M}}\right) & & =|\varphi|^{\mathcal{M}} \sqcup|\psi|^{\mathcal{M}} \\
|\varphi \rightarrow \psi|^{\mathcal{M}} & =|\varphi|^{\mathcal{M}} \Rightarrow|\psi|^{\mathcal{M}} & & \\
|\perp|^{\mathcal{M}} & =j \emptyset & & =\prod_{c \in U}|\varphi(c / v)|^{\mathcal{M}} \\
|\forall v \varphi|^{\mathcal{M}} & =\bigcap_{c \in U}|\varphi(c / v)|^{\mathcal{M}} & & =\bigsqcup_{c \in U}|\varphi(c / v)|^{\mathcal{M}} \\
|\exists v \varphi|^{\mathcal{M}} & =j\left(\bigcup_{c \in U}|\varphi(c / v)|^{\mathcal{M}}\right) & & \\
|\bigcirc \varphi|^{\mathcal{M}} & =\langle R\rangle|\varphi|^{\mathcal{M}} . &
\end{array}
$$


Here, $\sqcap, \sqcup, \sqcap, \sqcup$ refer to the meet and join operations in the complete lattice $\operatorname{Prop}(\mathcal{S})$. Now the definition of $|P|^{\mathcal{M}}$ ensures that $\left|P c_{1}, \ldots, c_{n}\right|^{\mathcal{M}} \in \operatorname{Prop}(\mathcal{S})$. In the case of $|\varphi \vee \psi|^{\mathcal{M}}$, if we assume that $|\varphi|^{\mathcal{M}}$ and $|\psi|^{\mathcal{M}}$ are propositions, then $|\varphi|^{\mathcal{M}} \cup|\psi|^{\mathcal{M}}$ is an up-set, hence $j\left(|\varphi|^{\mathcal{M}} \cup|\psi|^{\mathcal{M}}\right)$ is indeed the join $|\varphi|^{\mathcal{M}} \sqcup|\psi|^{\mathcal{M}}$. Similarly, $|\exists v \varphi|^{\mathcal{M}}$ is $\bigsqcup_{c \in U}|\varphi(c / v)|^{\mathcal{M}}$ if each $|\varphi(c / v)|^{\mathcal{M}}$ is a proposition. Then the closure properties of $\operatorname{Prop}(\mathcal{S})$ given in Theorem 3.5 and $(4.1)$ enable us to conclude, by induction on formula length, that the truth-set of any $\mathcal{L}^{U}$-sentence is a localised up-set:

Lemma 5.1 In any $Q L L$-model $\mathcal{M}$, every $\mathcal{L}^{U}$-sentence $\varphi$ has $|\varphi|^{\mathcal{M}} \in \operatorname{Prop}(\mathcal{S})$.

In particular, this means that satisfaction of a sentence is a local property, i.e. local satisfaction implies satisfaction:

$$
\text { if there exist an } x \text {-cover } C \subseteq|\varphi|^{\mathcal{M}} \text {, then } \mathcal{M}, x \mid=\varphi \text {. }
$$

Remark 5.2 The PLL-models of $[13,14]$ have an arbitrarily specified up-set $F$ of "fallible worlds", which serves as $|\perp|^{\mathcal{M}}$. A model is required to have $F \subseteq|\varphi|^{\mathcal{M}}$ in general, so that fallible worlds satisfy all sentences, ensuring the truth of $\perp \rightarrow \varphi$. In our present approach, such an $F$ is built into the cover system as the set $j \emptyset$ of points that have the empty cover.

A sentence $\varphi$ is said to be true in model $\mathcal{M}$, written $\mathcal{M} \models \varphi$, if it is true at every point, i.e. if $\mathcal{M}, x \models \varphi$ for all $x \in S$, or equivalently $|\varphi|^{\mathcal{M}}=S$. A formula $\varphi$ with free variables is true in $\mathcal{M}$ if every $\mathcal{L}^{U}$-sentence $\varphi\left(c_{1} / v_{1}, \ldots, c_{n} / v_{n}\right)$ that is a substitution instance of $\varphi$ is true in $\mathcal{M}$.

The following Lemma will be used below in proving the Soundness Theorem.

Lemma 5.3 For all $\mathcal{L}^{U}$-sentences $\varphi$ and $\psi, \mathcal{M} \models \varphi \rightarrow \psi$ iff $|\varphi|^{\mathcal{M}} \subseteq|\psi|^{\mathcal{M}}$. Hence $\mathcal{M} \models \varphi \leftrightarrow \psi$ iff $|\varphi|^{\mathcal{M}}=|\psi|^{\mathcal{M}}$.

We will also need the following simple fact, which is proven by induction on formula length.

Lemma 5.4 For any $\mathcal{L}^{U}$-formula $\varphi(v)$ with only $v$ free, and any constants $c, d$, if $|c|^{\mathcal{M}}=|d|^{\mathcal{M}}$ then $|\varphi(c / v)|^{\mathcal{M}}=|\varphi(d / v)|^{\mathcal{M}}$. In particular, this applies when $d=|c|^{\mathcal{M}} \in U$.

Theorem 5.5 (Soundness) If $Q L L \vdash \varphi$, then $\varphi$ is true in every $Q L L$-model $\mathcal{M}$.

Proof. Working within the model $\mathcal{M}$, we may drop the " $\mathcal{M}$ " and write satisfaction relations as " $x=\varphi$ ".

We have to show that if $\vdash \varphi$, then every sentential substitution instance $\varphi\left(c_{1} / v_{1}, \ldots, c_{n} / v_{n}\right)$ of $\varphi$ is true in $\mathcal{M}$. This is done as usual by induction, showing that the property holds when $\varphi$ is any axiom, and then that the inference rules preserve this property. But note that if $\varphi$ is any axiom, then $\varphi\left(c_{1} / v_{1}, \ldots, c_{n} / v_{n}\right)$ is also an axiom, of the same form. So for the base of the induction we show that every sentential axiom is true in $\mathcal{M}$.

Lemma 5.3 is used often to show $\mathcal{M} \models \varphi \rightarrow \psi$ by showing that $|\varphi|^{\mathcal{M}} \subseteq|\psi|^{\mathcal{M}}$, i.e. that $x \models \varphi$ implies $x \models \psi$ for an arbitrary $x$ in $\mathcal{M}$.

The proof that most of the axioms of non-modal intuitionistic propositional logic are true in $\mathcal{M}$ is straightforward, especially given the structure of $\operatorname{Prop}(\mathcal{S})$ as a Heyting algebra. For those such axioms not involving disjunction or $\perp$, the proof is as for Kripke's semantics.

For the cases involving $\vee$, note first that in the lattice $\operatorname{Prop}(\mathcal{S}),|\varphi|^{\mathcal{M}} \subseteq|\varphi|^{\mathcal{M}} \sqcup|\psi|^{\mathcal{M}}=|\varphi \vee \psi|^{\mathcal{M}}$, so $\varphi \rightarrow \varphi \vee \psi$ is true in $M$. Likewise for $\psi \rightarrow \varphi \vee \psi$.

For the axiom

$$
(\varphi \rightarrow \chi) \rightarrow((\psi \rightarrow \chi) \rightarrow(\varphi \vee \psi \rightarrow \chi))
$$

let $x \models(\varphi \rightarrow \chi)$ in order to show $x \models(\psi \rightarrow \chi) \rightarrow(\varphi \vee \psi \rightarrow \chi)$. Suppose that $x \leq y \models(\psi \rightarrow \chi)$. Then we have to show $y \models \varphi \vee \psi \rightarrow \chi$. So let $y \leq z \models \varphi \vee \psi$. Now we need $z \models \chi$.

Since $z=\varphi \vee \psi$, there exists a $z$-cover $C \subseteq|\varphi|^{\mathcal{M}} \cup|\psi|^{\mathcal{M}}$, hence $\uparrow C \subseteq|\varphi|^{\mathcal{M}} \cup|\psi|^{\mathcal{M}}$ as $|\varphi|^{\mathcal{M}} \cup|\psi|^{\mathcal{M}}$ is an up-set. By the localic condition, $C$ can be refined to an $z$-cover $C^{\prime}$ included in $\uparrow z$. Then any $c \in C^{\prime}$ has $z \leq c$, hence $x \leq y \leq c$. But $C^{\prime} \subseteq \uparrow C$, so either $c \models \varphi$, hence $c \models \chi$ as $x \models \varphi \rightarrow \chi$ and $x \leq c$; or else $c \models \psi$, hence again $c \models \chi$ as $y \models \psi \rightarrow \chi$ and $y \leq c$. Thus we have shown that $z \triangleleft C^{\prime} \subseteq|\chi|^{\mathcal{M}}$. Hence $z \models \chi$ as required, by the local character of satisfaction (5.2).

For the axiom $\perp \rightarrow \varphi$, observe that $|\perp|^{\mathcal{M}}$ is the least proposition $j \emptyset$ of $\mathcal{S}$, and so $|\perp|^{\mathcal{M}} \subseteq|\varphi|^{\mathcal{M}}$, giving $\mathcal{M}=\perp \rightarrow \varphi$. 
Now for the two quantifier axioms. For a sentence of the form $\forall v \varphi \rightarrow \varphi(d / v)$, by Lemma 5.4 we can assume $d \in U$. Then

$$
|\forall v \varphi|^{\mathcal{M}}=\bigcap_{c \in U}|\varphi(c / v)|^{\mathcal{M}} \subseteq|\varphi(d / v)|^{\mathcal{M}}
$$

making $\forall v \varphi \rightarrow \varphi(d / v)$ true in $\mathcal{M}$

For a sentence $\varphi(d / v) \rightarrow \exists v \varphi$, we have $|\varphi(d / v)|^{\mathcal{M}} \subseteq \bigsqcup_{c \in U}|\varphi(c / v)|^{\mathcal{M}}=|\exists v \varphi|^{\mathcal{M}}$.

The modal axioms are all true in $\mathcal{M}$ because $\langle R\rangle$ is a nucleus on $\operatorname{Prop}(\mathcal{S})$ (Lemma 4.1). Thus $|\varphi|^{\mathcal{M}} \subseteq\langle R\rangle|\varphi|^{\mathcal{M}}=|\bigcirc \varphi|^{\mathcal{M}}$, so $\mathcal{M} \models \varphi \rightarrow \bigcirc \varphi$. Similarly for the other two modal axioms.

That the rule of modus ponens preserves truth is well known, so we are left with the two quantifier rules. Consider a sentence $\varphi \rightarrow \forall v \psi$ with $v$ not free in $\varphi$, hence $(\varphi \rightarrow \psi)(c / v)=\varphi \rightarrow \psi(c / v)$. If $\varphi \rightarrow \psi$ is true in $\mathcal{M}$, then for all $c \in U, \mathcal{M}=\varphi \rightarrow \psi(c / v)$ and so $|\varphi|^{\mathcal{M}} \subseteq|\psi(c / v)|^{\mathcal{M}}$. Hence

$$
|\varphi|^{\mathcal{M}} \subseteq \bigcap_{c \in U}|\psi(c / v)|=|\forall v \psi|^{\mathcal{M}}
$$

so $\varphi \rightarrow \forall v \psi$ is true in $\mathcal{M}$.

Similarly, if $v$ is not free in $\psi$, and $\varphi \rightarrow \psi$ is true in $\mathcal{M}$, then $|\varphi(c / v)|^{\mathcal{M}} \subseteq|\psi|^{\mathcal{M}}$ for all $c \in U$, and

$$
|\exists v \varphi|^{\mathcal{M}}=\bigsqcup_{c \in U}|\varphi(c / v)|^{\mathcal{M}} \subseteq|\psi|^{\mathcal{M}},
$$

showing $\exists v \varphi \rightarrow \psi$ is true in $\mathcal{M}$.

\section{Completeness}

To prove that QLL is complete for truth in QLL-models, we carry out the standard Lindenbaum algebra construction, take the MacNeille completion of the Lindenbaum algebra, and then convert this into a QLL-model through the $\mathcal{S}_{\mathbf{H}}$ construction of Theorems 3.6 and 4.2.

Let $\mathcal{F}$ be the set of all $\mathcal{L}$-formulas, and $U_{\mathrm{t}}$ the set of all $\mathcal{L}$-terms. The condition " $-\varphi \rightarrow \psi$ and $\vdash \psi \rightarrow \varphi$ " defines an equivalence relation on $\mathcal{F}$. Let $\|\varphi\|$ be the equivalence class of $\varphi$, and $H^{\mathrm{QLL}}=$ $\{\|\varphi\|: \varphi \in \mathcal{F}\}$ the set of equivalence classes. A well-defined relation $\sqsubseteq$ on $H^{\mathrm{QLL}}$ is given by

$$
\|\varphi\| \sqsubseteq\|\psi\| \quad \text { iff } \quad \vdash \varphi \rightarrow \psi .
$$

This is a partial order, making $H^{\mathrm{QLL}}$ into a Heyting algebra $\mathbf{H}^{\mathrm{QLL}}$, the Lindenbaum algebra of $Q L L$, in which

$$
\begin{aligned}
\|\varphi\| \sqcap\|\psi\| & =\|\varphi \wedge \psi\| \\
\|\varphi\| \sqcup\|\psi\| & =\|\varphi \vee \psi\| \\
\|\varphi\| \Rightarrow\|\psi\| & =\|\varphi \rightarrow \psi\| \\
0 & =\|\perp\| \\
\|\forall v \varphi\| & =\prod_{\tau \in U_{\mathrm{t}}}\|\varphi(\tau / v)\| \\
\|\exists v \varphi\| & =\bigsqcup_{\tau \in U_{\mathrm{t}}}\|\varphi(\tau / v)\| .
\end{aligned}
$$

The greatest element of $\mathbf{H}^{\mathrm{QLL}}$ is characterised by

$$
\|\varphi\|=1 \quad \text { iff } \quad \vdash \varphi \text {. }
$$

Although $\mathbf{H}^{\mathrm{QLL}}$ has the stated meets and joins characterising $\|\forall v \varphi\|$ and $\|\exists v \varphi\|$ (cf., e.g., [5, $\left.\S 3.4\right]$ ), it is not a complete lattice. But if $\mathbf{H}$ is the MacNeille completion of $\mathbf{H}^{\mathrm{QLL}}$, then $\mathbf{H}^{\mathrm{QLL}}$ is a Heyting subalgebra of $\mathbf{H}$, and the inclusion $\mathbf{H}^{\mathrm{QLL}} \hookrightarrow \mathbf{H}$ preserves any existing meets and joins, so the equations of $(6.1)$ all remain true when read in $\mathbf{H}$. (6.2) is also true in $\mathbf{H}$, because it has the same greatest element as $\mathbf{H}^{\mathrm{QLL}}$.

Now QLL is closed under the monotonicity inference rule

$$
\frac{\varphi \rightarrow \psi}{\bigcirc \varphi \rightarrow \bigcirc \psi}
$$


from which it follows that if $\|\varphi\| \sqsubseteq\|\psi\|$ then $\|\bigcirc \varphi\| \sqsubseteq\|\bigcirc \psi\|$, so a well-defined modal operator $m$ on $\mathbf{H}^{\mathrm{QLL}}$ is given by

$$
m\|\varphi\|=\|\bigcirc \varphi\| \text {. }
$$

By the $\bigcirc$-axioms, this $m$ is a nucleus. By Corollary 2.6, the upper extension of $m$ is a nucleus on the McNeille completion $\mathbf{H}$ of $\mathbf{H}^{\mathrm{QLL}}$. We continue to refer to this extension as $m$, so can view (6.3) as a statement in $\mathbf{H}$. From $(\mathbf{H}, m)$ by Theorem 4.2 we obtain the nuclear localic modal cover system $\left(\mathcal{S}_{\mathbf{H}}, R_{m}\right)$ with $(\mathbf{H}, m)$ isomorphic to the modal locale $\left(\operatorname{Prop}\left(\mathcal{S}_{\mathbf{H}}\right),\left\langle R_{m}\right\rangle\right)$. Let $\theta: \mathbf{H} \rightarrow \operatorname{Prop}\left(\mathcal{S}_{\mathbf{H}}\right)$ be this isomorphism. Then we construct the model

$$
\mathcal{M}=\left(\mathcal{S}_{\mathbf{H}}, R_{m}, U_{\mathrm{t}},|\cdot|^{\mathcal{M}}\right),
$$

by taking the set $U_{\mathrm{t}}$ of $\mathcal{L}$-terms as the individuals of $\mathcal{M}$, and defining the interpretation $|\cdot|^{\mathcal{M}}$ by

$$
\begin{aligned}
|c|^{\mathcal{M}} & =c \in U_{\mathrm{t}}, \text { all } c \in \mathcal{L} ; \\
|P|^{\mathcal{M}}\left(\tau_{1}, \ldots, \tau_{n}\right) & =\theta\left\|P \tau_{1} \cdots \tau_{n}\right\| \in \operatorname{Prop}\left(\mathcal{S}_{\mathbf{H}}\right), \text { all } \tau_{1}, \ldots, \tau_{n} \in U_{\mathrm{t}} .
\end{aligned}
$$

Notice that every individual variable $v$, being an $\mathcal{L}$-term, is a member of $U_{\mathrm{t}}$, hence becomes a self-denoting

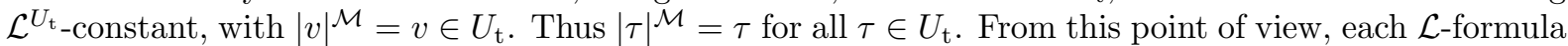
$\varphi$ becomes an $\mathcal{L}^{U_{\mathrm{t}}}$-sentence, and so has an interpretation as a proposition $|\varphi|^{\mathcal{M}} \in \operatorname{Prop}\left(\mathcal{S}_{\mathbf{H}}\right)$. We adopt this point of view in showing

Lemma 6.1 For any $\mathcal{L}$-formula $\varphi,|\varphi|^{\mathcal{M}}=\theta\|\varphi\|$.

Proof. By induction on formula length. If $\varphi$ is the atomic formula $P \tau_{1} \cdots \tau_{n}$, then

$$
|\varphi|^{\mathcal{M}}=|P|^{\mathcal{M}}\left(\left|\tau_{1}\right|^{\mathcal{M}}, \ldots,\left|\tau_{n}\right|^{\mathcal{M}}\right)=|P|^{\mathcal{M}}\left(\tau_{1}, \ldots, \tau_{n}\right),
$$

so the result holds by definition of $|P|^{\mathcal{M}}$. The inductive cases then hold from the equations (5.1) that express $|\varphi|^{\mathcal{M}}$ by the algebra of $\operatorname{Prop}\left(\mathcal{S}_{\mathbf{H}}\right)$, the equations $(6.1,6.3)$ that specify $\|\varphi\|$ by the algebra of $\mathbf{H}$, and the fact that the isomorphism $\theta$ preserves these equations. For instance, if the Lemma holds for $\varphi$, then

$$
|\bigcirc \varphi|^{\mathcal{M}}=\langle R\rangle|\varphi|^{\mathcal{M}}=\langle R\rangle \theta\|\varphi\|=\theta(m\|\varphi\|)=\theta\|\bigcirc \varphi\|,
$$

so it holds for $\bigcirc \varphi$. Similarly,

$$
|\forall v \varphi|^{\mathcal{M}}=\prod_{\tau \in U_{\mathrm{t}}}|\varphi(\tau / v)|^{\mathcal{M}}=\prod_{\tau \in U_{\mathrm{t}}} \theta\|\varphi(\tau / v)\|=\theta \prod_{\tau \in U_{\mathrm{t}}}\|\varphi(\tau / v)\|=\theta\|\forall v \varphi\|,
$$

and so on.

Theorem 6.2 (Completeness) If $\varphi$ is true in every $Q L L$-model, then $Q L L \vdash \varphi$.

Proof. Suppose $\varphi$ is true in every QLL-model. Let $\mathcal{M}$ be the QLL-model constructed above. Then $|\varphi|^{\mathcal{M}}=S$, so by Lemma $6.1, \theta\|\varphi\|=1$ in $\operatorname{Prop}\left(\mathcal{S}_{\mathbf{H}}\right)$. Hence $\|\varphi\|=1$ in $\mathbf{H}$, and so $\vdash \varphi$ by $(6.2)$.

Note that this proof, together with Soundness, shows that $\mathcal{M}$ is a single characteristic model for QLL, i.e. $\mathcal{M}=\varphi$ iff $\mathrm{QLL} \vdash \varphi$.

\section{Constructive $\mathrm{K}$ and $\mathrm{S} 4$}

Use of the MacNeille completion of a modal Heyting algebra, and its representation over a localic cover system, provides a general method for the analysis of intuitionistic modal logics by cover semantics. This is an alternative to the method of canonical models, which itself corresponds to the Stone representation of Heyting algebras using prime filters, and relates naturally to Kripke semantics with its classical treatment of disjunction and $\exists$.

For QLL we used the upper extension of a modal operator, because this preserves the property of being a nucleus. For other modalities the lower extension may be appropriate, while both extensions may be required for multi-modal logics. 


\begin{tabular}{|c|c|c|}
\hline \multicolumn{3}{|l|}{ Axioms } \\
\hline$\square \mathrm{K}: \quad \square(\varphi \rightarrow \psi) \rightarrow(\square \varphi \rightarrow \square \psi)$ & $\diamond \mathrm{K}:$ & $\square(\varphi \rightarrow \psi) \rightarrow(\diamond \varphi \rightarrow \diamond \psi)$ \\
\hline$\square \mathrm{T}: \quad \square \varphi \rightarrow \varphi$ & $\diamond \mathrm{T}:$ & $\varphi \rightarrow \diamond \varphi$ \\
\hline$\square 4: \quad \square \varphi \rightarrow \square \square \varphi$ & $\diamond 4:$ & $\diamond \nabla \varphi \rightarrow \diamond \varphi$ \\
\hline \multicolumn{3}{|l|}{$\begin{array}{l}\square \text {-Rule } \\
\varphi\end{array}$} \\
\hline
\end{tabular}

Figure 2: Modal Postulates for CS4

Cases in point are the system called CK, for Constructive $K$, in [36] and the system CS4, for Constructive $S_{4}$, of [3]. These have an intuitionistic propositional base, and a pair of modalities $\square, \diamond$. CS4 is defined by all the postulates of Figure 2, while CK has just the axioms $\square \mathrm{K}$ and $\diamond \mathrm{K}$ and the $\square$-rule. We define a $C K$-modal algebra $\left(\mathbf{H}, m_{\square}, m_{\diamond}\right)$ to comprise a Heyting algebra $\mathbf{H}$ carrying two modal operators $m_{\square}, m_{\diamond}$ such that

(1) $m_{\square}$ is multiplicative and satisfies $m_{\square} 1=1$, and

(2) $m_{\square} a \sqcap m_{\diamond} b \sqsubseteq m_{\diamond}(a \sqcap b)$.

If $\mathbf{H}$ is a locale, then we call this a CK-modal locale. A CS4-modal algebra is a CK-modal algebra in which $m_{\square}$ is also an interior operator and $m_{\diamond}$ is a closure operator (cf. [3, Def. 3]), and a CS4-modal locale is CS4-modal algebra for which $\mathbf{H}$ is a locale.

Note that the combination of $\square \mathrm{K}$ and the $\square$-rule is deductively equivalent over intuitionistic propositional logic to the combination of the axioms

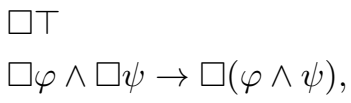

and the $\square$-monotonicity inference rule

$$
\frac{\varphi \rightarrow \psi}{\square \varphi \rightarrow \square \psi}
$$

This combination corresponds algebraically to the requirement that $m_{\square}$ be a modal operator satisfying (1) above. From $\diamond \mathrm{K}$ and the $\square$-rule we derive the $\diamond$-monotonicity inference rule

$$
\frac{\varphi \rightarrow \psi}{\diamond \varphi \rightarrow \diamond \psi}
$$

Also the theorem $\square \varphi \wedge \diamond \psi \rightarrow \diamond(\varphi \wedge \psi)$ is derivable in CK [36, Section 3]. These facts ensure that the Lindenbaum algebra of any logic that includes CK is a CK-modal algebra under the operators $m_{\square}\|\varphi\|=\|\square \varphi\|$ and $m_{\diamond}\|\varphi\|=\|\nabla \varphi\|$.

We define a $C K$-modal cover system to be a structure $\left(\mathcal{S}, R_{\square}, R_{\diamond}\right)$ with $\mathcal{S}$ being a localic cover system, and $R_{\square}, R_{\diamond}$ being binary relations on $\mathcal{S}$ each satisfying the Confluence and Modal Localisation properties, such that $R_{\square}$ is serial, i.e. $\forall x \exists y\left(x R_{\square} y\right)$, and the following hold:

(i) if $x R_{\square} y$ and $x R_{\square} z$ then for some $w \geq y, z$ we have $x R_{\square} w$.

(ii) if $x R_{\square} y$ and $x R_{\diamond} z$, then for some $w \geq y, z$ we have $x R_{\diamond} w$.

Condition (i) ensures that the axiom $\square \mathrm{K}$ is true in models on CK-modal cover systems, while (ii) ensures the truth of $\diamond \mathrm{K}$.

A CS4-modal cover system is a CK-modal cover system in which

(iii) $R_{\square}$ is a transitive subrelation of $\geq$, and

(iv) $R_{\diamond}$ is a reflexive and transitive.

These last two conditions ensure the truth of the remaining CS4 axioms. 
Theorem 7.1 If $\left(\mathcal{S}, R_{\square}, R_{\diamond}\right)$ is a CK-modal (respectively CS4-modal) cover system, then the algebra $\left(\operatorname{Prop}(\mathcal{S}),\left\langle R_{\square}\right\rangle,\left\langle R_{\diamond}\right\rangle\right)$ is a CK-modal (respectively CS4-modal) locale.

Proof. The Confluence and Modal Localisation properties ensure that $\left\langle R_{\square}\right\rangle$ and $\left\langle R_{\diamond}\right\rangle$ are both modal operators, i.e. monotone, on $\operatorname{Prop}(\mathcal{S})$.

Condition (i) ensures that $\left\langle R_{\square}\right\rangle X \cap\left\langle R_{\square}\right\rangle Y \subseteq\left\langle R_{\square}\right\rangle(X \cap Y)$ when $X, Y$ are up-sets, and hence that $\left\langle R_{\square}\right\rangle$ is multiplicative on $\operatorname{Prop}(\mathcal{S})$. The seriality condition $\forall x \exists y\left(x R_{\square} y\right)$ ensures that $S \subseteq\left\langle R_{\square}\right\rangle S$, hence $\left\langle R_{\square}\right\rangle 1=1$ in $\operatorname{Prop}(\mathcal{S})$. Thus (1) holds for $m_{\square}=\left\langle R_{\square}\right\rangle$.

Condition (ii) ensures that $\left\langle R_{\square}\right\rangle X \cap\left\langle R_{\diamond}\right\rangle Y \subseteq\left\langle R_{\diamond}\right\rangle(X \cap Y)$ when $X, Y$ are up-sets, so (2) holds for $m_{\diamond}=\left\langle R_{\diamond}\right\rangle$. Hence altogether $\left(\mathcal{S}, R_{\square}, R_{\diamond}\right)$ is a CK-modal cover system.

For the CS4 case, the conditions under (iii) ensure that $\left\langle R_{\square}\right\rangle$ is an interior operator, as follows. Transitivity of $R_{\square}$ makes $\left\langle R_{\square}\right\rangle\left\langle R_{\square}\right\rangle X \subseteq\left\langle R_{\square}\right\rangle X$. Having $R_{\square}$ a subrelation of $\geq$ makes $\left\langle R_{\square}\right\rangle$ deflationary, for if $x \in\left\langle R_{\square}\right\rangle X$, then for some $y, x R_{\square} y \in X$, so $x \geq y$, hence $x \in X$ if $X$ is an up-set. In particular, $\left\langle R_{\square}\right\rangle X \subseteq X$ for all propositions $X$, and altogether we have that $\left\langle R_{\square}\right\rangle$ is an interior operator. Finally, from (iv) it follows that $\left\langle R_{\diamond}\right\rangle$ is a closure operator, as mentioned in the proof of Lemma 4.1.

In the converse direction:

Theorem 7.2 If $\left(\mathbf{H}, m_{\square}, m_{\diamond}\right)$ is a CK-modal (respectively CS4-modal) locale, then $\left(\mathcal{S}_{\mathbf{H}}, R_{m_{\square}}, R_{m_{\diamond}}\right)$ is a CK-modal (respectively $\mathrm{CS}_{4}$-modal) cover system.

Proof. $\quad$ Recall that $x R_{m_{\square}} y$ iff $x \sqsubseteq m_{\square} y$, and $x R_{m_{\diamond}} y$ iff $x \sqsubseteq m_{\diamond} y$. The definition of $\mathcal{S}_{\mathbf{H}}$ itself was given in the proof of Theorem 3.6.

The Confluence and Modal Localisation properties hold for $R_{m_{\square}}$ and $R_{m_{\diamond}}$ just as for $R_{m}$ in the proof of Theorem 4.2, where the argument required only that $m$ was monotone. From $m_{\square} 1=1$ it follows that $x R_{m_{\square}} 1$ in general, so $R_{m_{\square}}$ is serial.

Now we need to show (i) and (ii) for $R_{\square}=R_{m_{\square}}$ and $R_{\diamond}=R_{m_{\diamond}}$.

(i): If $x R_{m_{\square}} y$ and $x R_{m_{\square}} z$, then $x \sqsubseteq m_{\square} y \sqcap m_{\square} z \sqsubseteq m_{\square}(y \sqcap z)$ by the multiplicativity of $m_{\square}$. Putting $w=y \sqcap z$ then gives $w \geq y, w \geq z$ and $x R_{m_{\square}} w$.

(ii): If $x R_{m_{\square}} y$ and $x R_{m_{\diamond}} z$, then $x \sqsubseteq m_{\square} y \sqcap m_{\diamond} z \sqsubseteq m_{\diamond}(y \sqcap z)$ by (2), so with $w=y \sqcap z$ we get $w \geq y, z$ and $x R_{m_{\diamond}} w$.

For the CS4 case, we need to show (iii) and (iv) for $R_{m_{\square}}$ and $R_{m_{\diamond}}$, given that $m_{\square}$ is also an interior operator and $m_{\diamond}$ is a closure operator.

(iii): $R_{m_{\square}}$ is transitive because $m_{\square} m_{\square} x \sqsubseteq m_{\square} x$ (as in Theorem 4.2), and $R_{m_{\square}}$ is a subrelation of $\geq$, because if $x \sqsubseteq m_{\square} y$ then $x \sqsubseteq y$ as $m_{\square} y \sqsubseteq y$, and this means that $x \geq y$.

(iv): $R_{m_{\diamond}}$ is a reflexive and transitive when $m_{\diamond}$ is a closure operator, as in the proof of Theorem 4.2.

The logic QCK is defined axiomatically, in a first-order language with $\square$ and $\diamond$, by the standard axiomatisation of first-order intuitionistic predicate logic, together with the axioms $\square \mathrm{K}$ and $\diamond \mathrm{K}$ and the $\square$-rule. QCS4 has all the axioms and rules of QCK and also the other CS4 axioms of Figure 2.

Now let L be any logical system whose axioms and rules include those of QCK. Let $\left(\mathbf{H}^{\mathrm{L}}, m_{\square}, m_{\diamond}\right)$ be the Lindenbaum algebra of L, with $m_{\square}\|\varphi\|=\|\square \varphi\|$ and $m_{\diamond}\|\varphi\|=\|\nabla \varphi\|$, and let $\left(\mathbf{H}, m_{\square}^{\circ}, m_{\diamond}^{\bullet}\right)$ be its MacNeille completion, defined to comprise the MacNeille completion $\mathbf{H}$ of $\mathbf{H}^{\mathrm{L}}$ with the lower extension of $m_{\square}$ and the upper extension of $m_{\diamond}$. This Lindenbaum algebra is a CK-modal algebra. Hence by Theorem 2.3, $m_{\square}^{\circ}$ is a multiplicative operator. It satisfies $m_{\square}^{\circ} 1=1$, since $m_{\square} 1=1$ and $\mathbf{H}^{\mathrm{L}}$ and $\mathbf{H}$ have the same greatest element. By Theorem 2.5, $m_{\square}^{\circ} x \sqcap m_{\diamond}^{\bullet} \sqsubseteq m_{\diamond}^{\bullet}(x \sqcap y)$. Moreover, if L includes the CS4 axioms, then $m_{\square}$ is an interior operator, hence by Corollary $2.4 m_{\square}^{\circ}$ is an interior operator; and $m_{\diamond}$ is a closure operator, hence by Theorem $2.2 m_{\diamond}^{\bullet}$ is a closure operator.

In particular, these observations establish the following:

Theorem 7.3 The MacNeille completion of the Lindenbaum algebra of QCK (respectively QCS4) is a QCK-modal (respectively QCS4-modal) locale.

This provides a basis for developing a model theory for QK and QCS4 using cover systems, adapting our methodology for QLL. A $Q C K$-model has the form $\mathcal{M}=\left(\mathcal{S}, R_{\square}, R_{\diamond}, U,|\cdot|^{\mathcal{M}}\right)$, where $\left(\mathcal{S}, R_{\square}, R_{\diamond}\right)$ is a CK-modal cover system, and $U$ and $|\cdot|^{\mathcal{M}}$ are as for QLL-models. The satisfaction relation is defined as before, except that we now have

$$
\begin{aligned}
& \mathcal{M}, x \models \square \varphi \quad \text { iff } \quad \text { for some } y, x R_{\square} y \text { and } \mathcal{M}, y=\varphi, \\
& \mathcal{M}, x \models \nabla \varphi \quad \text { iff } \quad \text { for some } y, x R_{\diamond} y \text { and } \mathcal{M}, y \models \varphi .
\end{aligned}
$$


A QCS4-model is a QCK-model whose underlying cover system is CS4-modal, i.e. satisfies (iii) and (iv) above. We leave it to the reader to put together the results of this section to show

\section{Theorem 7.4 (Soundness and Completeness)}

(1) $Q C K \vdash \varphi$ iff $\varphi$ is true in all QCK-models.

(2) $Q C S_{4} \vdash \varphi$ iff $\varphi$ is true in all QCS4-models.

The models for CK and CS4 of [36, 3] have a single binary relation $R$ which is used to interpret $\square$ and $\diamond$ by the semantics

$$
\begin{aligned}
& \mathcal{M}, x \models \square \varphi \quad \text { iff } \quad \forall y \geq x \forall z(y R z \text { implies } \mathcal{M}, z \models \varphi), \\
& \mathcal{M}, x \models \nabla \varphi \quad \text { iff } \quad \forall y \geq x \exists z(y R z \text { and } \mathcal{M}, z \models \varphi) .
\end{aligned}
$$

The non-modal propositional connectives are treated as in Kripke's intuitionistic semantics (except that $|\perp|^{\mathcal{M}}$ may be a non-empty set of fallible worlds - see Remark 5.2). Here we have seen that if the cover semantics is used instead, then both $\square$ and $\diamond$ can be interpreted as existential quantifiers bounded by two relations, as in (7.1), that are interconnected by (ii) above. So they are both treated somewhat like Boolean diamonds. This underscores the point that there is more to intuitionistic modal logic than combining the classical semantic analysis of $\square$ and $\diamond$ with non-modal intuitionistic models.

We can also extend this approach to provide a cover semantics for the system of first-order intuitionistic modal logic studied in [45]. This can be defined by adding to QCK the axiom $\neg \diamond \perp$, i.e. $\diamond \perp \rightarrow \perp,{ }^{11}$ and was shown in [45] to be characterised by models that combine Kripke's semantics for first-order intuitionistic logic with the interpretation (7.2) of $\square$ and $\diamond$. These models have no fallible worlds, i.e. have $|\perp|^{\mathcal{M}}=\emptyset$.

The axiom $\neg \diamond \perp$ corresponds algebraically to the requirement that $m_{\diamond} 0=0$, an equation that holds in the Lindenbaum algebra of any logic in which $\neg \diamond \perp$ is a theorem. Moreover, in general $m_{\diamond} 0=0$ implies $m_{\diamond}^{\bullet} 0=0$, so the equation is preserved by upper MacNeille completion. Model-theoretically, truth of $\neg \diamond \perp$ in $\mathcal{M}$ requires that $|\nabla \perp|^{\mathcal{M}}=|\perp|^{\mathcal{M}}$, which in a model on a CK-modal cover system $\left(\mathcal{S}, R_{\square}, R_{\diamond}\right)$ requires that $\left\langle R_{\diamond}\right\rangle j \emptyset=j \emptyset$. Since $j \emptyset$ is invariably the least member of $\operatorname{Prop}(\mathcal{S})$, this is equivalent to $\left\langle R_{\diamond}\right\rangle j \emptyset \subseteq j \emptyset$, which means that

(v) $x R_{\diamond} y \triangleleft \emptyset$ implies $x \triangleleft \emptyset$.

Now if $\left(\mathbf{H}, m_{\square}, m_{\diamond}\right)$ is a CK-modal locale having $m_{\diamond} 0=0$, then in $\mathcal{S}_{\mathbf{H}}$, condition (v) holds when $R_{\diamond}=R_{m_{\diamond}}$. For if $x R_{m_{\diamond}} y \triangleleft \emptyset$, then $x \sqsubseteq m_{\diamond} y=m_{\diamond}(\bigsqcup \emptyset)=m_{\diamond} 0=0$, hence $x=\bigsqcup \emptyset$ and so $x \triangleleft \emptyset$. Combining these observations with our general methodology leads to the conclusion that

$$
\text { QCK }+\neg \diamond \perp \vdash \varphi \text { iff } \varphi \text { is true in all QCK-models that satisfy (v). }
$$

This characterisation can be refined a little further. We noted just before Corollary 3.7 that in the cover system $\mathcal{S}_{\mathbf{H}}$ of any locale $\mathbf{H}$, the set $j \emptyset$ is a singleton (namely $\{0\}$ ). Thus whenever we give a completeness theorem by using the cover system associated with the MacNeille completion of a Lindenbaum algebra, we obtain a characterisation of the logic in question by models in which there is exactly one member that has the empty cover and so satisfies $\perp$. Logics containing $\neg \diamond \perp$ are characterised by models in which there is an element $\infty$ such that

$$
\left\langle R_{\diamond}\right\rangle j \emptyset=j \emptyset=\{\infty\} .
$$

This amounts to requiring that for all members $x$,

$$
x R_{\diamond} \infty \quad \text { iff } \quad x \triangleleft \emptyset \quad \text { iff } \quad x=\infty .
$$

In particular, the logic QCK $+\neg \diamond \perp$ is characterised by the class of all QCK-models that have a unique such empty-covered "fallible" point $\infty$ satisfying $\diamond \perp$ and $\perp$.

\footnotetext{
${ }^{11}$ The system of [45] also has $\square \varphi \wedge \diamond(\varphi \rightarrow \psi) \rightarrow \diamond \psi$ as an axiom, but this is derivable in CK, as shown in [36], and is readily seen, with the help of condition (ii), to be true in all of our models based on CK-modal cover systems.
} 


\section{Non-Modal First-Order Intuitionistic Predicate Logic}

Apart from the analysis of modalities, our results provide a completeness theorem for first-order intuitionistic logic itself with respect to cover semantics. That is not itself a new result (see $[43, \S 13.7]$ for instance), but there is interest in alternative model constructions that lead to the characterisation, and here we sketch an alternative to the algebraic methods used above that were based on the MacNeille completion. We can use instead a construction in the Henkin style, building a model whose points are certain sets of formulas, with these sets having closure properties determined by the proof theory of the logic. Since we are not using the classical semantics for disjunction, we do not have to make our formulasets "prime", i.e. they do not have to contain one of $\varphi$ and $\psi$ whenever they contain $\varphi \vee \psi$. Nor do they need to have the "saturation", or " $\exists$-witnessing" property of containing some instantiation $\varphi(\tau / v)$ whenever they contain $\exists v \varphi$. So we are able to make a simpler and more explicit model construction than the Henkin models introduced for Kripke semantics, as in [1, 42, 15].

We now outline this construction. Throughout this section, a "formula" will mean any $\bigcirc$-free $\mathcal{L}$ formula as defined in Section 5, and the notation " $\vdash$ " will mean that $\varphi$ is a theorem of first-order intuitionistic logic, i.e. $\varphi$ is derivable using the $\bigcirc$-free axioms and rules from Figure 1. We also write $\varphi \vdash \psi$ when $\vdash \varphi \rightarrow \psi$. We will assume many of the basic properties of this deducibility relation $\vdash$ for quantified intuitionistic logic.

A model for the $\bigcirc$-free language has the form $\mathcal{M}=(\mathcal{S}, U,|\cdot| \mathcal{M})$, with $\mathcal{S}$ a localic cover system, and is defined just like the notion of a QLL-model, but without the relation $R$ or any of the properties associated with $R$. From the proof of Theorem 5.5 (Soundness), we can take it as given that every theorem of first-order intuitionistic logic is true in all models on localic cover systems.

For each $\varphi$, let $\varphi^{\vdash}=\{\psi: \varphi \vdash \psi\}$. A set of the form $\varphi^{\vdash}$ will be called a principal theory. Note that $\varphi_{1}^{\vdash} \subseteq \varphi_{2}^{\vdash}$ iff $\varphi_{2} \vdash \varphi_{1}$, and that $\varphi_{1}^{\vdash}=\varphi_{2}^{\vdash}$ iff $\vdash \varphi_{1} \leftrightarrow \varphi_{2}$. Amongst the closure properties of principal theories that are readily demonstrated are:

$$
\begin{aligned}
& \text { Conjunction : } \psi_{1} \wedge \psi_{2} \in \varphi^{\vdash} \text { iff } \psi_{1} \in \varphi^{\vdash} \text { and } \psi_{2} \in \varphi^{\vdash} \text {; } \\
& \text { Deduction : if } \psi_{1} \in \varphi^{\vdash} \text { and } \psi_{1} \vdash \psi_{2} \text { then } \psi_{2} \in \varphi^{\vdash} ; \\
& \text { Modus Ponens }: \text { if } \psi_{1}, \psi_{1} \rightarrow \psi_{2} \in \varphi^{\vdash} \text {, then } \psi_{2} \in \varphi^{\vdash} .
\end{aligned}
$$

Define $\mathcal{S}_{p}=\left(S_{p}, \leq, \triangleleft\right)$, the principal system, by

- $S_{p}=\{x: x$ is a principal theory $\}$;

- $x \leq y$ iff $x \subseteq y$;

- $x \triangleleft C$ iff $x=\bigcap C$, where $C \subseteq S_{p}$.

Lemma $8.1 \mathcal{S}_{p}$ is a strictly localic cover system.

Proof. We check the cover system axioms.

Existence: Put $C=\{x\}$. Then $x \triangleleft C \subseteq \uparrow x$. (Alternatively, put $C=\uparrow x$.)

Transitivity: if $x=\bigcap C$ and for all $y \in C, y=\bigcap C_{y}$, then by set theory $x=\bigcap\left(\bigcup_{y \in C} C_{y}\right)$.

Refinement: let $x \leq y$ and $C \triangleright x$. We have to show that $C$ can be refined to some $y$-cover $C^{\prime}$. Let $x=\varphi^{\vdash}$ and $y=\psi^{\vdash}$, and define $C^{\prime}=\left\{(\psi \wedge \chi)^{\vdash}: \chi^{\vdash} \in C\right\}$. Then as $\chi^{\vdash} \subseteq(\psi \wedge \chi)^{\vdash}$, we get $C^{\prime} \subseteq \uparrow C$, i.e. $C^{\prime}$ refines $C$. It remains to show that $C^{\prime}$ is a $y$-cover.

Now as $y=\psi^{\vdash} \subseteq(\psi \wedge \chi)^{\vdash}$, we have $y \subseteq \bigcap C^{\prime}$. But if $\rho \in \bigcap C^{\prime}$, then for each $\chi^{\vdash} \in C$ we have $\rho \in(\psi \wedge \chi)^{\vdash}$, so $\psi \wedge \chi \vdash \rho$, hence $\chi \vdash \psi \rightarrow \rho$, and thus $\psi \rightarrow \rho \in \chi^{\vdash}$. This shows that $\psi \rightarrow \rho \in \bigcap C$. But $\bigcap C=x \subseteq y$, and $\psi \in y$, so $\rho \in y$ by Modus Ponens (8.3). Thus we have $y=\bigcap C^{\prime}$, i.e. $y \triangleleft C^{\prime}$ as required.

Finally, as $x=\bigcap C$ implies $C \subseteq\{y: x \subseteq y\}=\uparrow x$, the system $\mathcal{S}_{p}$ is strictly localic.

Note that $\mathcal{S}_{p}$ shares with the locale-based systems $\mathcal{S}_{\mathbf{H}}$ the property that $j \emptyset$ is a singleton. The principal theory

$$
(\perp)^{\vdash}=\{\psi: \perp \vdash \psi\}
$$

is in fact the set of all formulas, hence is equal to $\bigcap \emptyset$, and so is the unique member of $\mathcal{S}_{p}$ that is covered by the empty set. 
For each formula $\varphi$, define $|\varphi|=\left\{x \in S_{p}: \varphi \in x\right\}$. We will define a model $\mathcal{M}_{p}$ on $\mathcal{S}_{p}$ in which $|\varphi|$ is the truth set $|\varphi|^{\mathcal{M}_{p}}$. As before, this model will use the set $U_{\mathrm{t}}$ of $\mathcal{L}$-terms as its domain of individuals, and will be a single characteristic model for the logic. For this we first show that membership of $|\varphi|$ behaves like the satisfaction relation:

Lemma 8.2 In the principal cover system $\mathcal{S}_{p}$, for any $x \in S_{p}$ :

(1) $\varphi \wedge \psi \in x$ iff $\varphi \in x$ and $\psi \in x$.

(2) $\varphi \vee \psi \in x$ iff there is some $C \subseteq S_{p}$ with $x \triangleleft C \subseteq|\varphi| \cup|\psi|$.

(3) $\varphi \rightarrow \psi \in x$ iff for all $y \supseteq x, \varphi \in y$ implies $\psi \in y$.

(4) $\perp \in x$ iff $\emptyset \triangleright x$ iff $x$ is the set of all formulas.

(5) $\forall v \varphi \in x$ iff for all terms $\tau \in U_{\mathrm{t}}, \varphi(\tau / v) \in x$.

(6) $\exists v \varphi \in x$ iff there is some $C \subseteq S_{p}$ with $x \triangleleft C \subseteq \bigcup_{\tau \in U_{\mathrm{t}}}|\varphi(\tau / v)|$.

Proof. $\quad$ For each $y \in S_{p}$, fix a formula $\varphi_{y} \in y$ such that $y=\left(\varphi_{y}\right)^{\vdash}=\left\{\psi: \varphi_{y} \vdash \psi\right\}$.

(1): This was noted in (8.1).

(2): Let $\varphi \vee \psi \in x$. Put $C=\left|\varphi_{x}\right| \cap(|\varphi| \cup|\psi|) \subseteq|\varphi| \cup|\psi|$. It suffices to show $x \triangleleft C$. First, if $y \in C$, then $\varphi_{x} \in y$, so $\varphi_{y} \vdash \varphi_{x}$, hence $\left(\varphi_{x}\right)^{\vdash} \subseteq\left(\varphi_{y}\right)^{\vdash}$, i.e. $x \subseteq y$. Thus $x \subseteq \bigcap C$. But if $\rho \in \bigcap C$, then as $\left(\varphi_{x} \wedge \varphi\right)^{\vdash} \in\left|\varphi_{x}\right| \cap|\varphi| \subseteq C$ we get $\varphi_{x} \wedge \varphi \vdash \rho$. Similarly, $\varphi_{x} \wedge \psi \vdash \rho$. Hence $\left(\varphi_{x} \wedge \varphi\right) \vee\left(\varphi_{x} \wedge \psi\right) \vdash \rho$. So by distributivity $\varphi_{x} \wedge(\varphi \vee \psi) \vdash \rho$. But $\varphi_{x}, \varphi \vee \psi \in x$, so $\varphi_{x} \wedge(\varphi \vee \psi) \in x$ (8.1), hence $\rho \in x$ (8.2). This shows $x=\bigcap C$ as required.

Conversely, suppose some $C$ has $x \triangleleft C \subseteq|\varphi| \cup|\psi|$. By the axiom $\varphi \rightarrow \varphi \vee \psi,|\varphi| \subseteq|\varphi \vee \psi|$. Similarly $|\psi| \subseteq|\varphi \vee \psi|$. Hence $C \subseteq|\varphi \vee \psi|$. Thus $\varphi \vee \psi \in \bigcap|\varphi \vee \psi| \subseteq \bigcap C=x$, so $\varphi \vee \psi \in x$.

(3): If $\varphi \rightarrow \psi \in x$, then if $\varphi \in y \supseteq x$, we get $\psi \in y$ by Modus Ponens (8.3). Conversely, if $\varphi \in y \supseteq x$ implies $\psi \in y$, let $y=\left(\varphi_{x} \wedge \varphi\right)^{\vdash}$ to conclude that $\varphi_{x} \wedge \varphi \vdash \psi$. Hence $\varphi_{x} \vdash \varphi \rightarrow \psi$, giving $\varphi \rightarrow \psi \in x$.

(4): Let $\Phi$ be the set of all formulas. Using the axiom $\perp \rightarrow \varphi$, we get $\perp \in x$ iff $x=\Phi$. But $\emptyset \triangleright x$ iff $x=\bigcap \emptyset$ iff $x=\Phi$.

(5): If $\forall v \varphi \in x$, then $\varphi(\tau / v) \in x$ by the axiom $\forall v \varphi \rightarrow \varphi(\tau / v)$ and Modus Ponens. Conversely, assuming $\varphi(\tau / v) \in x$ for all $\tau$, by choosing some variable $w$ not occuring in $\varphi_{x}$ or $\varphi$ we get $\varphi_{x} \vdash \varphi(w / v)$, hence $\varphi_{x} \vdash \forall w \varphi(w / v)$ by the inference rule for $\forall$. But $\forall w \varphi(w / v) \vdash \forall v \varphi$, leading to $\varphi_{x} \vdash \forall v \varphi$, hence $\forall v \varphi \in x$.

(6): Let $\exists v \varphi \in x$. Put

$$
C=\left|\varphi_{x}\right| \cap \bigcup_{\tau \in U_{\mathrm{t}}}|\varphi(\tau / v)|=\bigcup_{\tau \in U_{\mathrm{t}}}\left|\varphi_{x} \wedge \varphi(\tau / v)\right|
$$

Since $C \subseteq \bigcup_{\tau \in U_{\mathrm{t}}}|\varphi(\tau / v)|$, it suffices to show $x \triangleleft C$. Now if $y \in C$, then $\varphi_{x} \in y$, so $x \subseteq y$ as in the proof of (2). Thus $x \subseteq \bigcap C$. But if $\rho \in \bigcap C$, then as $\left(\varphi_{x} \wedge \varphi(\tau / v)\right)^{\vdash} \in\left|\varphi_{x} \wedge \varphi(\tau / v)\right| \subseteq C$ we get $\varphi_{x} \wedge \varphi(\tau / v) \vdash \rho$ for all $\tau \in U_{\mathrm{t}}$. Now choose a variable $w$ that does not occur in any of $\varphi_{x}, \varphi$ or $\rho$. Then from $\varphi_{x} \wedge \varphi(w / v) \vdash \rho$ we get $\exists w\left(\varphi_{x} \wedge \varphi(w / v)\right) \vdash \rho$ as $w$ is not free in $\rho$. But $\varphi_{x} \wedge \exists w \varphi(w / v) \vdash \exists w\left(\varphi_{x} \wedge \varphi(w / v)\right)$ as $w$ is not free in $\varphi_{x}$, so this implies $\varphi_{x} \wedge \exists w \varphi(w / v) \vdash \rho$. Now $\exists v \varphi \vdash \exists w \varphi(w / v)$, and we have $\exists v \varphi \in x$, so $\exists w \varphi(w / v) \in x$, hence $\varphi_{x} \wedge \exists w \varphi(w / v) \in x$ as also $\varphi_{x} \in x$. But then $\rho \in x$. This completes the required proof that $x=\bigcap C$.

Finally, for the converse direction of (6), suppose there exists $C$ with $x \triangleleft C \subseteq \bigcup_{\tau \in U_{\mathrm{t}}}|\varphi(\tau / v)|$. From the axiom $\varphi(\tau / v) \rightarrow \exists v \varphi$ we have $|\varphi(\tau / v)| \subseteq|\exists v \varphi|$ for all $\tau$, so $C \subseteq|\exists v \varphi|$. Thus $\exists v \varphi \in \bigcap|\exists v \varphi| \subseteq \bigcap C=$ $x$, so $\exists v \varphi \in x$.

Now define the desired principal model $\mathcal{M}_{p}=\left(\mathcal{S}_{p}, U_{\mathrm{t}},|\cdot|^{\mathcal{M}_{p}}\right)$ on $\mathcal{S}_{p}$ by putting

$$
\begin{aligned}
|c|^{\mathcal{M}_{p}} & =c \in U_{\mathrm{t}}, \text { all } c \in \mathcal{L} \\
|P|^{\mathcal{M}_{p}}\left(\tau_{1}, \ldots, \tau_{n}\right) & =\left|P \tau_{1} \cdots \tau_{n}\right|=\left\{x \in S: P \tau_{1} \cdots \tau_{n} \in x\right\}, \text { all } \tau_{1}, \ldots, \tau_{n} \in U_{\mathrm{t}} .
\end{aligned}
$$

As with the model of Section 6 , we view each $\mathcal{L}$-formula $\varphi$ as an $\mathcal{L}^{U_{\mathrm{t}}}$-sentence, with $|\tau|^{\mathcal{M}_{p}}=\tau$ for all $\tau \in U_{\mathrm{t}}$.

Lemma 8.3 For any $\varphi$ and any $x \in S_{p}, \mathcal{M}_{p}, x \mid=\varphi$ iff $\varphi \in x$. In other words, $|\varphi|^{\mathcal{M}_{p}}=|\varphi|$. 
Proof. By induction on formula length. For the atomic case,

$$
\left|P \tau_{1} \cdots \tau_{n}\right|^{\mathcal{M}_{p}}=|P|^{\mathcal{M}_{p}}\left(\left|\tau_{1}\right|^{\mathcal{M}}, \ldots,\left|\tau_{n}\right|^{\mathcal{M}}\right)=|P|^{\mathcal{M}_{p}}\left(\tau_{1}, \ldots, \tau_{n}\right)=\left|P \tau_{1} \cdots \tau_{n}\right|
$$

by definition of $|\cdot|^{\mathcal{M}_{p}}$. The inductive cases then all follow using Lemma 8.2.

We get from this that $\mathcal{M}_{p}$ is a characteristic model for intuitionistic first-order logic. But further, we obtain a special point $x_{\top}$ of $\mathcal{M}_{p}$ such that theoremhood is characterised by truth at $x_{\top}$. Let $\top$ be the sentence $\perp \rightarrow \perp$. Then in general $\vdash \varphi$ iff $\vdash \top \rightarrow \varphi$ iff $\varphi \in T^{\vdash}$. Putting $x_{\top}=T^{\vdash}$, we have

Theorem 8.4 For any formula $\varphi$,

(1) $\mathcal{M}_{p} \models \varphi$ iff $\mathcal{M}_{p}, x_{\top} \models \varphi$ iff $\vdash \varphi$.

(2) $\varphi$ is a theorem of first-order intuitionistic logic iff $\varphi$ is true in all models based on (strictly) localic cover systems.

Proof.

(1) If $\mathcal{M}_{p} \models \varphi$ then $\mathcal{M}_{p}, x_{\top} \models \varphi$, hence $\varphi \in x_{\top}$ by Lemma 8.3 , which implies $\vdash \varphi$ as noted above. But if $\vdash \varphi$, then $\varphi$ is true in all models on localic cover systems (cf. the Soundness Theorem 5.5), hence true in $\mathcal{M}_{p}$.

(2) From (1) and Soundness.

In conclusion we make three observations about this principal model construction. First, an algebraic version of the construction gives a representation of an arbitrary Heyting algebra $\mathbf{H}$ as an algebra of propositions over a strictly localic cover system (cf. Corollary 3.7). This representation does not depend on any set-theoretic principles needed that ensure the existence of prime filters, as in the Stone representation. For each $a \in \mathbf{H}$, the set $[a)=\{b \in \mathbf{H}: a \sqsubseteq b\}$ is the principal filter of $\mathbf{H}$ generated by $a$. Let $\mathcal{S}_{\mathbf{H}}^{p}$ be the system based on the set of all principal filters of $\mathbf{H}$, with $x \leq y$ iff $x \subseteq y$, and $x \triangleleft C$ iff $x=\bigcap C$. Adapting the above arguments shows that $\mathcal{S}_{\mathbf{H}}^{p}$ is a strictly localic cover system, and $a \mapsto\{x: a \in x\}$ is an injective homomorphism from $\mathbf{H}$ into $\operatorname{Prop}\left(\mathcal{S}_{\mathbf{H}}^{p}\right)$ that preserves any joins and meets that exist in $\mathbf{H}$.

Second, the reader may wonder whether we could have used this construction to prove the completeness of QLL for its cover semantics. A natural binary relation $R_{\bigcirc}$ on $S_{p}$ can be defined from $\bigcirc$ by putting $x R_{\bigcirc} y$ iff $\varphi_{x} \vdash \bigcirc \varphi_{y}$, where $\varphi_{x}, \varphi_{y}$ are chosen generators of $x, y$ as in the proof of Theorem 8.2. Then it can be shown that

$$
\bigcirc \varphi \in x \text { iff for some } y, x R_{\bigcirc} y \text { and } \varphi \in y \text {, }
$$

so $|\bigcirc \varphi|=\left\langle R_{\odot}\right\rangle|\varphi|$ and membership of $|\bigcirc \varphi|$ behaves like satisfaction for $\bigcirc$. Moreover, $R_{\bigcirc}$ is confluent and nuclear. But the stumbling block is the Modal Localisation property, which does not appear to be provable for $R_{\bigcirc}$. It is for that reason that we resorted to the use of the MacNeille completion to construct models that have this essential property.

Thirdly, this principal model construction supports the informational interpretation of the cover relation $\triangleright$ mentioned at the beginning of Section 3. If the points of $\mathcal{S}$ are taken to be states containing certain information, with $x \models \varphi$ iff $x$ contains the information that $\varphi$, then $C \triangleright x$ iff the information content of $x$ comprises the information that is common to all the members of $C$.

\section{References}

[1] Peter Aczel. Saturated intuitionistic theories. In H. Arnold Schmidt, K. Schütte, and H.-J. Thiele, editors, Contributions to Mathematical Logic. Proceedings of the Logic Colloquium, Hannover 1966, pages 1-11. North-Holland, 1968.

[2] Peter Aczel. The Russell-Prawitz modality. Mathematical Structures in Computer Science, 11(4):541-554, 2001. 
[3] Natasha Alechina, Michael Mendler, Valeria de Paiva, and Eike Ritter. Categorical and Kripke semantics for constructive S4 modal logic. In Laurent Fribourg, editor, Computer Science Logic: 15th International Workshop, CSL 2001, volume 2142 of Lecture Notes in Computer Science, pages 292-307. Springer-Verlag, 2001.

[4] J. L. Bell. Toposes and Local Set Theories. Oxford University Press, 1988.

[5] J. L. Bell and A. B. Slomson. Models and Ultraproducts. North-Holland, Amsterdam, 1969.

[6] John L. Bell. Cover schemes, frame-valued sets and their potential uses in spacetime physics. In Albert Reimer, editor, Spacetime Physics Research Trends, Horizons in World Physics, volume 248. Nova Science Publishers, 2005. Manuscript at http://publish.uwo.ca/ jbell.

[7] P. N. Benton, G. M. Bierman, and V. C. V. de Paiva. Computational types from a logical perspective. Journal of Functional Programming, 8:177-193, 1998.

[8] E. W. Beth. Semantic construction of intuitionistic logic. Medededlingen der koninklijke Nederlandse Akademie van Wetenschappen, afd. Letterkunde. Nieuwe Reeks, 19(11):357-388, 1956.

[9] Haskell B. Curry. The elimination theorem when modality is present. The Journal of Symbolic Logic, 17:249-265, 1952.

[10] B. A. Davey and H. A. Priestley. Introduction to Lattices and Order. Cambridge University Press, 1990.

[11] Leo Esakia. The modalized Heyting calculus: a conservative modal extension of the Intuitionistic Logic. Journal of Applied Non-Classical Logics, 16(3-4):349-366, 2006.

[12] M. Fairtlough and M. Walton. Quantified lax logic. Technical Report CS-97-11, Department of Computer Science, University of Sheffield, July 1997.

[13] Matt Fairtlough and Michael Mendler. An intuitionistic modal logic with applications to the formal verification of hardware. In Leszek Pacholoski and Jerzy Tiuryn, editors, Computer Science Logic. Proceedings 1994, volume 933 of Lecture Notes in Computer Science, pages 354-368. Springer-Verlag, 1995.

[14] Matt Fairtlough and Michael Mendler. Propositional lax logic. Information and Computation, 137:1-33, 1997.

[15] M. C. Fitting. Intuitionistic Logic, Model Theory \& Forcing. North-Holland, 1969.

[16] M. P. Fourman and D. S. Scott. Sheaves and logic. In M. P. Fourman, C. J. Mulvey, and D. S. Scott, editors, Applications of Sheaves, volume 753 of Lecture Notes in Mathematics, pages 302-401. Springer-Verlag, 1979.

[17] Deepak Garg and Martin Abadi. A modal deconstruction of access control logics. In Roberto M. Amadio, editor, Foundations of Software Science and Computational Structures, 11th International Conference, FOSSACS 2008, volume 4962 of Lecture Notes in Computer Science, pages 16-230. Springer-Verlag, 2008.

[18] Deepak Garg and Michael Carl Tschantz. From indexed lax logic to intuitionistic logic. Technical Report CMU-CS-07-167, School of Computer Science, Carnegie Mellon University, January 2008. www.cs.cmu.edu/ dg/publications.html.

[19] S. Givant and Y. Venema. The preservation of Sahlqvist equations in completions of Boolean algebras with operators. Algebra Universalis, 41:47-84, 1999.

[20] Robert Goldblatt. Grothendieck topology as geometric modality. Zeitschrift für Mathematische Logik und Grundlagen der Mathematik, 27:495-529, 1981. Reprinted in [21].

[21] Robert Goldblatt. Mathematics of Modality. CSLI Lecture Notes No. 43. CSLI Publications, Stanford University, 1993. 
[22] Robert Goldblatt. A Kripke-Joyal semantics for noncommutative logic in quantales. In Guido Governatori, Ian Hodkinson, and Yde Venema, editors, Advances in Modal Logic, Volume 6, pages 209-225. College Publications, London, 2006. www.aiml .net/volumes/volume6/.

[23] Robert Goldblatt. Mathematical modal logic: A view of its evolution. In Dov M. Gabbay and John Woods, editors, Logic and the Modalities in the Twentieth Century, volume 7 of Handbook of the History of Logic, pages 1-98. Elsevier, 2006. Manuscript available at www.msor.vuw.ac.nz/ rob.

[24] Robert Goldblatt. Topoi. The Categorial Analysis of Logic. Dover Publications, Inc., Mineola, New York, 2006.

[25] Robert Goldblatt and Ian Hodkinson. Commutativity of quantifiers in varying-domain Kripke models. In David Makinson, Jacek Malinowski, and Heinrich Wansing, editors, Towards Mathematical Philosophy, volume 28 of Trends in Logic, pages 9-30. Springer, 2009.

[26] P. T. Johnstone. Topos Theory. Academic Press, New York, 1977.

[27] P. T. Johnstone. Stone Spaces. Cambridge University Press, 1982.

[28] Anders Kock. Universal projective geometry via topos theory. Journal of Pure and Applied Algebra, 9:1-24, 1976.

[29] Saul A. Kripke. Semantical analysis of intuitionistic logic I. In J. N. Crossley and M. A. E. Dummett, editors, Formal Systems and Recursive Functions, pages 92-130. North-Holland, 1965.

[30] A. V. Kuznetsov and A. Yu. Muravitsky. On superintuitionistic logics as fragments of proof logic extensions. Studia Logica, 45(1):77-99, 1986.

[31] J. Lambek and P. J. Scott. Introduction to Higher Order Categorical Logic. Cambridge University Press, 1986.

[32] Saunders Mac Lane and Ieke Moerdijk. Sheaves in Geometry and Logic: A First Introduction to Topos Theory. Springer-Verlag, 1992.

[33] F. W. Lawvere. Quantifiers and sheaves. Actes des Congrès International des Mathématiques, 1:329-334, 1970.

[34] D. S. Macnab. Modal operators on Heyting algebras. Algebra Universalis, 12:5-29, 1981.

[35] H. M. MacNeille. Partially ordered sets. Transactions of the American Mathematical Society, 42:416460, 1937.

[36] Michael Mendler and Valeria de Paiva. Constructive CK for contexts. In Luciano Serafini and Paolo Bouquet, editors, Proceedings of the CRR '05 Workshop on Context Representation and Reasoning, Paris, France, July 5, 2005. http://sunsite.informatik.rwth-aachen.de/Publications/ CEUR-WS/Vol-136/

[37] Eugenio Moggi. Notions of computation and monads. Information and Computation, 93:55-92, 1991.

[38] James R. Munkres. Topology. Prentice Hall, 2000.

[39] Krister Segerberg. Propositional logics related to Heyting's and Johansson's. Theoria, 34:26-61, 1968.

[40] M. H. Stone. Topological representations of distributive lattices and Brouwerian logics. Casopis pro Pestovani Matematiky a Fysiky, 67:1-25, 1937-8.

[41] Mark Theunissen and Yde Venema. MacNeille completions of lattice expansions. Algebra Universalis, 57:143-193, 2007.

[42] Richmond H. Thomason. On the strong semantical completeness of the intuitionistic predicate calculus. The Journal of Symbolic Logic, 33(1):1-7, 1968.

[43] A. S. Troelstra and D. van Dalen. Constructivism in Mathematics: An Introduction II, volume 123 of Studies in Logic. North-Holland, 1988. 
[44] A. S. Troelstra and P. van Ulsen. The discovery of E. W. Beth's semantics for intuitionistic logic. In Jelle Gerbrandy, Maarten Marx, Maarten de Rijke, and Yde Venema, editors, JFAK. Essays Dedicated to Johan van Benthem on the Occasion of his 50th Birthday, Vossiuspers. Amsterdam University Press, 1999. ISBN 905629104 1, www.illc.uva.nl/j50/contribs/troelstra/.

[45] Duminda Wijesekera. Constructive modal logics I. Annals of Pure and Applied Logic, 50:271-301, 1990. 